Research paper

\title{
The CREp program and the ICE-D production rate calibration database: A fully parameterizable and updated online tool to compute cosmic-
} ray exposure ages

\author{
L.C.P. Martin ${ }^{\text {a, }}$ P.-H. Blard ${ }^{\text {a, }}{ }^{*}$, G. Balco ${ }^{\text {b }}$, J. Lavé ${ }^{\text {a }}$, R. Delunel ${ }^{\text {c }}$, N. Lifton ${ }^{\text {d, }}$, V. Laurent ${ }^{\text {a }}$ \\ ${ }^{a}$ Centre de Recherches Pétrographiques et Géochimiques, CRPG, UMR 7358, CNRS, Université de Lorraine, Vandoeuvre-lès-Nancy, France \\ ${ }^{\mathrm{b}}$ Berkeley Geochronological Center, BGC, Berkeley, CA, USA \\ ${ }^{\mathrm{c}}$ Institute of Geological Sciences, University of Bern, Bern, Switzerland \\ d Purdue University, West Lafayette, IN, USA
}

\section{A R T I C L E I N F O}

\section{Article history:}

Received 8 June 2016

Received in revised form

17 October 2016

Accepted 30 November 2016

Available online 3 December 2016

\section{Keywords:}

Cosmic-ray exposure age computation

ICE-D database

Cosmogenic ${ }^{10} \mathrm{Be}$ and ${ }^{3} \mathrm{He}$

Scaling model

Atmosphere model

Geomagnetic reconstruction model

Production rate parameterization

\begin{abstract}
A B S T R A C T
Over the last decades, cosmogenic exposure dating has permitted major advances in many fields of Earth surface sciences and particularly in paleoglaciology. Yet, exposure age calculation remains a complicated and dense procedure. It requires numerous choices of parameterization and the use of an accurate production rate.

This study describes the CREp program (http://crep.crpg.cnrs-nancy.fr) and the ICE-D production rate online database (http://calibration.ice-d.org). This system is designed so that the CREp calculator will automatically reflect the current state of this global calibration database production rate, ICE-D. ICE-D will be regularly updated in order to incorporate new calibration data and reflect the current state of the available literature.

CREp is a Octave/Matlab ${ }^{\odot}$ online code that computes Cosmic Ray Exposure (CRE) ages for ${ }^{3} \mathrm{He}$ and ${ }^{10} \mathrm{Be}$. A stand-alone version of the CREp code is also released with the present article. Note however that only the online version is connected to the online database ICE-D. The CREp program offers the possibility to calculate ages with two scaling models: i.e. the empirical Lal-Stone time-dependent model (Balco et al., 2008; Lal, 1991; Stone, 2000) with the muon parameters of Braucher et al. (2011), and the Lifton-SatoDunai (LSD) theoretical model (Lifton et al., 2014). The default atmosphere model is the ERA-40 database (Uppala et al., 2005), but one may also use the standard atmosphere for comparison (N.O.A.A. 1976). To perform the time-dependent correction, users may import their own geomagnetic database for paleomagnetic corrections or opt for one of the three proposed datasets (Lifton, 2016; Lifton et al., 2014; Muscheler et al., 2005).

For the important choice of the production rate, CREp is linked to a database of production rate calibration data that is part of the ICE-D (Informal Cosmogenic-nuclide Exposure-age Database) project (http://calibration.ice-d.org). This database includes published empirical calibration rate studies that are publicly available at present, comprising those of the CRONUS-Earth and CRONUS-EU projects, as well as studies from other projects. In the present study, the efficacy of the different scaling models has also been evaluated looking at the statistical dispersion of the computed Sea Level High Latitude (SLHL) production rates. Lal/Stone and LSD models have comparable efficacies, and the impact of the tested atmospheric model and the geomagnetic database is also limited.

Users however have several possibilities to select the production rate: 1) using a worldwide mean value, 2) a regionally averaged value (not available in regions with no data), 3) a local unique value, which can be chosen among the existing dataset or imported by the user, or 4) any combination of multiple calibration data.

If a global mean is chosen, the $1 \sigma$ uncertainty arising from the production rate is about $5 \%$ for ${ }^{10} \mathrm{Be}$ and $10 \%$ for ${ }^{3} \mathrm{He}$. If a regional production rate is picked, these uncertainties are potentially lower.
\end{abstract}

\footnotetext{
* Corresponding author. CRPG, 15 rue Notre Dame des Pauvres, 54500, Vandoeuvre-lès-Nancy, France.

E-mail addresses: leom@laposte.net (L.C.P. Martin), blard@crpg.cnrs-nancy.fr (P.-H. Blard), balcs@bgc.org (G. Balco).
} 
CREp is able to calculate a large number of ages in a reasonable time (typically $<30 \mathrm{~s}$ for 50 samples). The user may export a summary table of the computed ages and the density probability function associated with each age (in the form of a spreadsheet).

(c) 2016 Elsevier B.V. All rights reserved.

\section{Introduction}

\subsection{Cosmic-rays exposure age calculation}

Over the last decades, the development of Cosmic Ray Exposure dating method has permitted major advances in many fields of Earth sciences, notably in paleoglaciology (e.g. Barrows et al., 2011; Blard et al., 2007; Gosse et al., 1995; Jomelli et al., 2014; Licciardi et al., 2009; Smith et al., 2005) tectonics (e.g. Palumbo et al., 2004; Ritz et al., 1995), paleoaltimetry (e.g. Blard et al., 2006) landscape evolution (e.g. Brown et al., 1995; Riebe et al., 2000) or sediment transfer in the floodplain (e.g. Lupker et al., 2012; Wittmann and von Blanckenburg, 2009).

The calculation of accurate and precise CRE ages requires an accurate knowledge of the production rate of the cosmogenic nuclide in a specific mineral at a place to be dated, over the time frame of interest. Yet, in most cases, this local production rate is computed from calibration sites that may be located hundreds or thousands of kilometres away from the studied place, on objects that are sometimes significantly older or younger than the dated surface. As production rates vary with latitude, altitude, and also with time due to temporal fluctuations in the atmospheric density and the Earth's magnetic field, a complicated scaling procedure is necessary to obtain the appropriate production rate for dating. The usual method consists of, first, converting the reference production rate at a calibration site into a Sea Level High Latitude (SLHL) rate, corrected for the past geomagnetic activity over the calibration time period. Then, this SLHL production rate is rescaled to the location of the surface to be dated (Balco et al., 2008; Gosse and Phillips, 2001). For calculation consistency, these two transformations must be done using the same scaling scheme. This scaling scheme implies a model describing spatial variation in the cosmic ray flux, an atmosphere model, a reconstruction of the past geomagnetic activity, and an accurate model linking this paleomagnetic activity with the production of cosmogenic nuclides (Balco et al., 2008; Lifton et al., 2014; Dunai, 2001). Therefore, exposure age computation is not unique and the choices of the scaling framework and corresponding reference production rate may significantly impact the computed ages.

\subsection{Published calculation software and recent advances in CRE dating}

Over the last twenty years, several programs have been published to enable the calculations required to compute exposure ages: the WebCN online program (Ma et al., 2007), the Cosmocalc Excel $^{\odot}$ add-in (Vermeesch, 2007), the CRONUS-Earth online calculators (Balco et al., 2008; Marrero et al., 2016), the ACE Pythonbased software (Zweck et al., 2012) and other tools (Phillips et al., 2001; Schimmelpfennig et al., 2011). All of these tools are based on different approaches.

Yet, since the publication of these programs, the cosmogenic nuclide community has produced new developments and results that have improved the accuracy of exposure ages. Importantly, several new production rate calibration studies are now published each year, improving the method's accuracy in several regions.
Incorporating this new information into an exposure-age calculation scheme represents a challenge in data assimilation.

The default SLHL production rate used in the original CRONUS calculator for ${ }^{10} \mathrm{Be}$ is a global average deduced from several calibration sites $\left(\sim 4.5\right.$ at $\mathrm{g}^{-1} \mathrm{yr}^{-1}$ with the Lm scaling, description in Section 2.2 of Balco et al., 2008). However, recent calibration studies yielded significantly lower SLHL ${ }^{10} \mathrm{Be}$ production rates ( $<4$ at $\mathrm{g}^{-1} \mathrm{yr}^{-1}$ for the same scaling scheme) (e.g. Fenton et al., 2011; Kaplan et al., 2011; Putnam et al., 2010; Stroeven et al., 2015; Young et al., 2013; Martin et al., 2015). Moreover, statistical analyses highlighted the imperfections of scaling schemes to fit the production rates in certain regions, notably at high elevations low latitudes (Borchers et al., 2016; Lifton et al., 2014). This observation tends to suggest that the use of local calibration rate is more pertinent to compute accurate ages. Indeed, using a production rate derived from a calibration site that is both close in space and time to the site to date lowers the dependence of the production rate value to the scaling procedure (Balco et al., 2008). In this regard, the ongoing enrichment of the worldwide database of production rate is likely to efficiently improve the accuracy of CRE dating. Our opinion is that an online calculator connected to a regularly updated database is the most efficient means to achieve this goal.

The new theoretical LSD model and the associated publication (Lifton et al., 2014) brought important insights on the inaccuracies of former scaling models. Neutron-monitor based models (Desilets and Zreda, 2003; Desilets et al., 2006; Dunai, 2001; Lifton et al., 2005) are prone to overestimate the altitude dependence of cosmogenic-nuclide production while the older Lal-Stone model turned out to be robust and in good agreement with the LSD model. This is due to the multiplicity effect affecting neutron monitor data: this bias is indeed larger at high cutoff rigidities, for altitude above $3 \mathrm{~km}$ (Lifton et al., 2014). Borchers et al. (2016) confirmed these observations: using a least square criterion, they showed that the Lal-Stone models (with and without geomagnetic time corrections) and the LSD models are much more efficient than neutron-monitor based models (Desilets and Zreda, 2003; Dunai, 2001; Lifton et al., 2005 ) in predicting the spatial variability of production rates. With the LSD model, Lifton et al. (2014) also came up with a new 2D atmosphere model derived from the ERA-40 meteorological reanalysis (Uppala et al., 2005). Similarly to the NCEP/NCAR 2-D reanalysis (Balco et al., 2008), ERA-40 provides a globally consistent alternative to the independently determined pre-existing local atmosphere parameters (e.g. Farber et al., 2005 for the Andean atmosphere).

The impact of geomagnetic variations on cosmogenic nuclide production rates may be accounted for using various reconstructions of the geomagnetic activity. So far, the initial CRONUS-Earth calculator (Balco et al., 2008) relies on a composite framework including the Yang et al. (2000), Korte et al. (2005) and SINT800 (Guyodo and Valet, 1999) reconstructions, whereas the Lifton et al. (2014) LSD model uses CALS3k.3 (Korte et al., 2009), CALS7k.2 (Korte et al., 2005), GLOPIS-75 (Laj et al., 2004) and PADM2M (Ziegler et al., 2011). Yet Martin et al. (2015), Delunel et al. (2016) and Lifton (2016) showed that the choice of the geomagnetic database is likely to influence the ability of a scaling scheme to fit a set of production rates to SLHL values. 
1.3. The CREp program and the ICE-D production rate calibration database

The production rate and the scaling computation have thus a critical importance on the accuracy of the computed exposure ages. Therefore, it is crucial for the users to be able to test various production rates among the most relevant values and to perform sensitivity tests on the scaling choices using the most efficient models. For this purpose, we present the CREp program, an Octave/ Matlab ${ }^{\oplus}$ program that allows exposure ages computation for ${ }^{3} \mathrm{He}$ and ${ }^{10} \mathrm{Be}$ using a large choice of parameterization possibilities. CREp is linked with the ICE-D production rate calibration database, a new online database that compiles all existing calibration studies. This innovation makes CREp a flexible and easily updatable online calculator.

\section{Description of CREp}

CREp exists in the form of a Matlab ${ }^{\odot}$ code released as supplementary material of the present article. An Octave online version of CREp is also available at crep.crpg.cnrs-nancy.fr. The online version should be favoured, since it will incorporate the most recent developments, notably the most up-to-date production rates of the ICE-D database.

\subsection{Synoptic flow of CREp}

The global synoptic flow of CREp can be summarized in four steps (Fig. 1). First, user chooses the scaling procedure (scaling scheme, atmosphere, geomagnetic database). Second, user enters the characteristics of the samples to be dated (nuclide concentrations and uncertainties, geographical coordinates, elevation, sample thickness, density, potential erosion). Third, a specific production rate is chosen among several possibilities, those ranging from a global average to a single local calibration site. Finally, the exposure ages of the loaded samples are calculated with this production rate.

\subsection{Scaling models}

The CREp program includes two scaling models, the so-called Lm ("Lal modified", Balco et al., 2008) and the LSD model (Lifton et al., 2014). The Lal-Stone modified model corresponds to a time dependent version of the classical Lal-Stone scaling model that relies on nuclear emulsions, cloud chambers, and various neutron detectors for measurement of secondary cosmic-ray fluxes (Lal, 1991; Stone, 2000). In order to implement the time-dependent geomagnetic correction, the fundamental equations of Lal-Stone have been modified to use cutoff-rigidities rather than latitude as input, following Balco et al. (2008). For this, the model uses the equation linking the cutoff rigidity $R_{c}(G V)$ and the latitude (Elsasser et al., 1956; Stormer, 1955):

$$
R_{e}=\frac{M \mu_{0} c}{16 \pi \times 10^{9} r^{2}} \cos ^{4} \lambda
$$

where $\mathrm{M}\left(\mathrm{A} \mathrm{m}^{2}\right)$ is moment of the Earth dipole field, $\mathrm{r}$ is the mean radius of the Earth $\left(6.3712 \times 10^{6} \mathrm{~m}\right), \mathrm{c}$ is the speed of light $\left(3.0 \times 10^{8} \mathrm{~m} \mathrm{~s}^{-1}\right)$, and $\mu_{0}$ is the permeability of free space $\left(4 \pi \times 10^{-7} \mathrm{~N} \mathrm{~A}^{-2}\right)$. Using the 2010 value for $\mathrm{M}$ $\left(\mathrm{M}_{0}=7.746 \times 10^{22} \mathrm{~A} \mathrm{~m}^{2}\right)$, Equation (1) reduces to (Lifton et al., 2008):

$R_{e}=14.3 \frac{M}{M_{0}} \cos ^{4} \lambda$
For certain periods of the past, larger values of $\mathrm{M}$ imply cutoff rigidities larger than $14.3 \mathrm{GV}$. In that case, we assume a log-log interpolation between $R_{c}$ and the nucleon flux determined by the parameters of Lal-Stone for $R_{c}$ values ranging between 14.3 and 25 GV (Sandstrom et al., 1962).

In CREp, the spatial scaling of muogenic production is done using the parameters of Stone (2000) but includes the empirical muogenic/spallogenic production ratios determined by Braucher et al. (2011) (Table 1). This was motivated by several lines of evidence indicating that the parameters of Heisinger et al. (2002a,b) overestimated the muogenic production (Braucher et al., 2013, 2011; Kim and Englert, 2004).

The recently published LSD model (Lifton et al., 2014) derives scaling factors from integrals of neutron and proton fluxes, calculated using the PHITS-based Analytical Radiation Model in the Atmosphere (PARMA model; Sato et al., 2008), in conjunction with the most up to date neutron and proton excitation functions. In its original version, the LSD model proposes to either compute scaling factors for spallation only, or to take into account specific cross sections for each particle, nuclide and target element. The LSD version implemented in CREp only considers this second approach and computes the spatial scaling with specific cross sections for each nuclide $\left({ }^{10} \mathrm{Be}\right.$ and $\left.{ }^{3} \mathrm{He}\right)$. Consequently, ${ }^{3} \mathrm{He}$ and ${ }^{10} \mathrm{Be}$ follow different spatial scaling laws with this LSD model (see Fig. 8 in Lifton et al. (2014)).

LSD and Lal/Stone were the two retained models because they presently show the best ability to describe the evolution of production rates in time and space relatively to the other models based on neutron-monitor data (Section 1.2) (Borchers et al., 2016).

\subsection{Atmosphere models}

Two atmospheric models are proposed in the CREp program. First, the ERA-40 atmosphere 2D model, based on a re-analysis of meteorological data over 45 years between 1957 and 2002 produced by the European Centre for Medium-Range Weather Forecasts (Uppala et al., 2005). The ERA-40 database provides a local atmospheric pressure on any location of the Earth surface. ERA-40 notably describes quite accurately the local peculiarities of the present-day pressure field. The CREp program uses the Lifton et al. (2014) implementation of this atmospheric dataset, at a spatial resolution of $125 \mathrm{~km}$. This implemented version of ERA-40 is a combination of a sea level pressure and a $1000 \mathrm{hPa}$ temperature grids, used to compute the pressure at any elevation.

Alternatively, users may choose to use the U.S. Standard Atmosphere 1D model (N.O.A.A, 1976), that computes the pressure $\mathrm{P}(\mathrm{z})$ at an altitude $\mathrm{z}$, according to:

$P(z)=P_{S} \exp \left\{-\frac{g M}{R}\left[\ln T_{S}-\ln \left(T_{S}-z\right)\right]\right\}$

with sea level pressure $\mathrm{P}_{\mathrm{S}}=1013.25 \mathrm{hPa}$, sea level temperature $\mathrm{T}_{\mathrm{S}}=288.15 \mathrm{~K}$, and a standard lapse rate $\xi=0.0065 \mathrm{~K} \mathrm{~m}^{-1} \mathrm{~g}$ is the gravity acceleration, $\mathrm{R}$ the gas constant, and $\mathrm{M}$ the molar weight of air, yielding $\mathrm{gM} / \mathrm{R}=0.03417 \mathrm{~K} \mathrm{~m}^{-1}$.

It is worth noting that this $1 \mathrm{D}$ model does not take into account the local peculiarities of the atmospheric pressure field and may lead to significant biases in some particular regions, such as Antarctica (Stone, 2000). CREp users should thus use this atmosphere model with caution.

\subsection{Geomagnetic databases}

To account for the impact of the past geomagnetic activity on the production, CREp allows choosing between different 


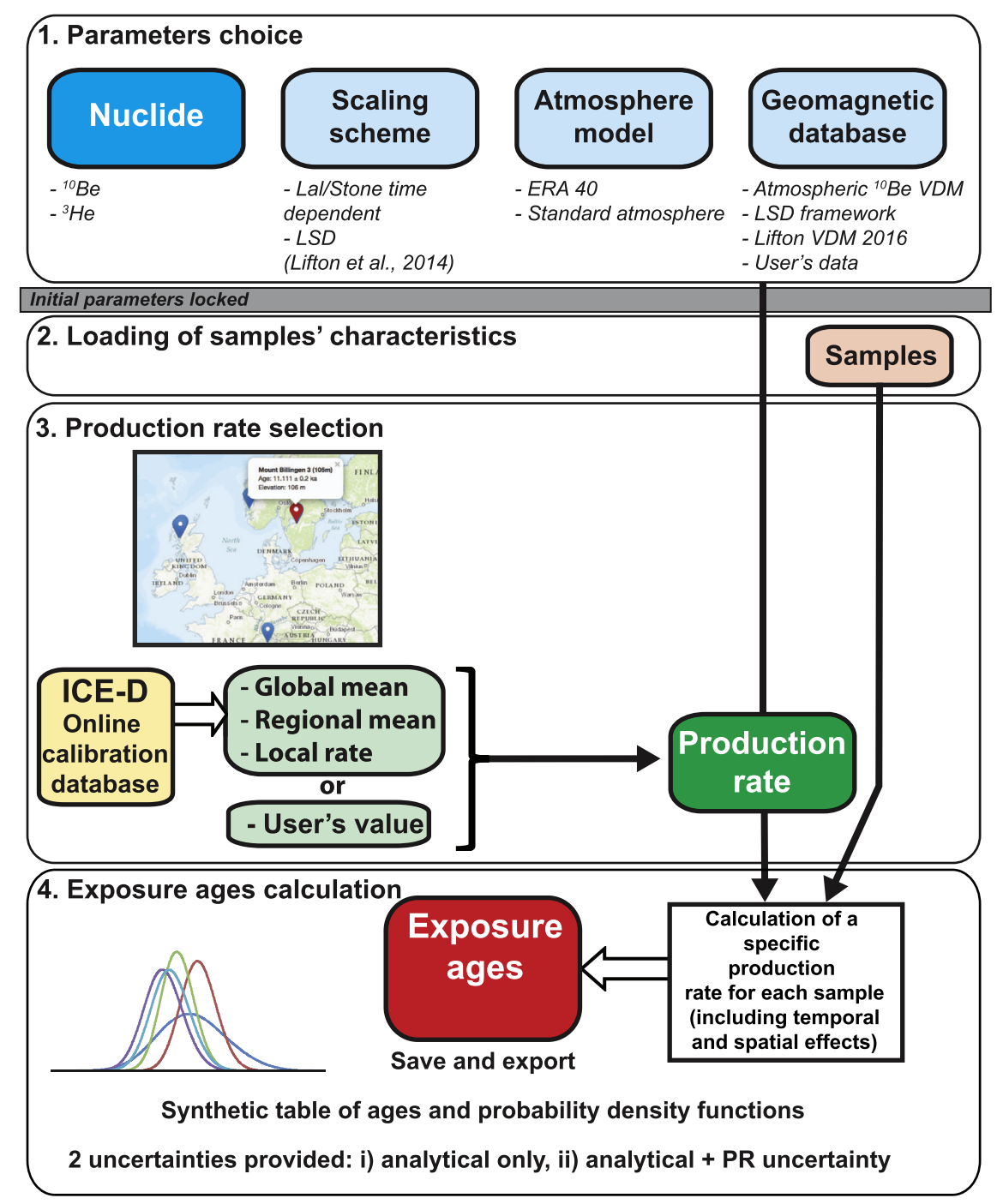

Fig. 1. Synoptic flow diagram of the CREp program.

Table 1

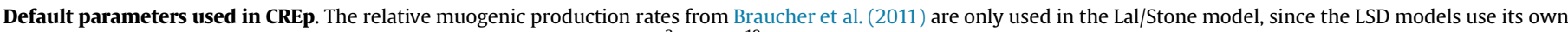
muon and neutron scaling procedure. Parameters are the same for both ${ }^{3} \mathrm{He}$ and ${ }^{10} \mathrm{Be}$.

\begin{tabular}{|c|c|c|c|c|}
\hline Parameters & Symbol & Value & Unit & Reference \\
\hline Fast muon relative production rate at sea level high latitude & - & 0.87 & $\%$ of the total production & Braucher et al., 2011 \\
\hline Slow muon relative production rate at sea level high latitude & - & 0.27 & $\%$ of the total production & Braucher et al., 2011 \\
\hline Attenuation length for spallation in rock/soil & $\Lambda$ & 160 & $\mathrm{~g} \mathrm{~cm}^{-2}$ & Gosse and Phillips, 2001 \\
\hline Density (Only used when it is not provided) & $\rho$ & 2.7 & $\mathrm{~g} \mathrm{~cm}^{-3}$ & Average value of continental crustal rocks \\
\hline Dipolar moment of the Earth magnetic field in 2010 & $\mathrm{M}_{0}$ & $7.746 \times 1022$ & $\mathrm{~A} \mathrm{~m} \mathrm{~m}^{2}$ & Finlay et al., 2010 \\
\hline
\end{tabular}

geomagnetic records or to import any original Virtual Axial Dipole Moment (VADM) dataset. The origin of the data implemented in the software and their associated parameterization in the scaling models are summarized in Table 2.

The three proposed geomagnetic databases included in CREp are: (i) the atmospheric ${ }^{10} \mathrm{Be}$-based VADM (Muscheler et al., 2005), ii) the geomagnetic framework initially published in the LSD model (Lifton et al., 2014) (a composite database that combines the geomagnetic reconstructions of the CALS7k.2 and CALS3k.3 model for the 0-7 ka BP period (Korte et al., 2009, 2005), the GLOPIS-75 reconstruction for the 7 to18 $\mathrm{ka} B P$ period and the PADM2M reconstruction (Ziegler et al., 2011) from 18 ka to 2 Ma BP), and iii) the recent composite VDM/VADM record proposed by Lifton (2016). All the original publications used to build these original geomagnetic records are given in Table 2. Few adjustments have been done to include these databases in CREp: the Muscheler et al. (2005) and the GLOPIS-75 databases are tuned to the GICC05 timescale (Svensson et al., 2008) using the g2n Matlab ${ }^{\circledR}$ function from Obrochta et al. (2014). To improve the computation speed, the Muscheler et al. (2005) record has been interpolated on a 50 years time step. For ages older than $60 \mathrm{ka}$, this reconstruction has been extended with the SINT-2000 record with a 1 kyr time step. The Lifton (2016) composite VDM/VADM has a varying time step but the average value for the last $200 \mathrm{ka}$ is $200 \mathrm{yr}$. The LSD framework 
Table 2

Geomagnetic reconstructions used in the scaling models of the CREp program.

\begin{tabular}{|c|c|c|c|}
\hline Geomagnetic reconstruction & Reference & Period & Geomagnetic dataset \\
\hline Atmospheric $10 \mathrm{Be}$-based $\mathrm{VDM}^{\mathrm{a}}$ & Muscheler et al. (2005) & $\begin{array}{l}0-60 \mathrm{ka} \\
60-2000 \mathrm{ka}\end{array}$ & $\begin{array}{l}\text { Muscheler et al., } 2005 \\
\text { Valet et al., } 2005\end{array}$ \\
\hline LSD framework & Lifton et al. (2014) & $\begin{array}{l}0-7 \mathrm{ka} \\
7-18 \mathrm{ka} \\
18-2000 \mathrm{ka}\end{array}$ & $\begin{array}{l}\text { CALS7k.2 and CALS3k.3 (Korte et al., 2005; Korte et al., 2009) } \\
\text { GLOPIS-75 (Laj et al., 2004) } \\
\text { PADM2M (Ziegler et al., 2011) }\end{array}$ \\
\hline Lifton 2016 VDM & Lifton (2016) & $\begin{array}{l}0-14 \mathrm{ka} \\
14-75 \mathrm{ka} \\
75-2000 \mathrm{ka}\end{array}$ & $\begin{array}{l}\text { Pavón-Carrasco et al., } 2014 \\
\text { GLOPIS-75 (Laj et al., 2004) } \\
\text { PADM2M (Ziegler et al., 2011) }\end{array}$ \\
\hline
\end{tabular}

a VDM: Virtual Dipolar Moment. All the dipolar moments are normalized to the present value of $7.746 \times 1022 \mathrm{~A}$ m-2 (Finlay et al., 2010 ).

b Spatialized cutoff rigidity used in the LSD model (Lifton et al., 2014) and global cutoff rigidity derived from the VDM for the "Lal modified" model (Balco et al., 2008; Lal, 1991; Stone, 2000).

presents a time step of $100 \mathrm{yr}$ until $50 \mathrm{ka}$ and $1 \mathrm{kyr}$ beyond.

If the LSD scaling model is selected with the LSD geomagnetic framework, the CREp calculator uses the LSD Matlab ${ }^{\odot}$ code of Lifton et al. (2014) in its original form. The "Lal modified" model and the LSD model each use different geomagnetic inputs for the $0-7 \mathrm{ka}$ period: while the "Lal modified" model always uses the $\mathrm{M} / \mathrm{M}_{0}$ ratio (the VDM or VADM normalized to the value in 2010), the LSD model only uses it beyond $7 \mathrm{ka}$ BP. Between 0 and $7 \mathrm{ka}$ BP, the LSD model directly uses globally gridded cutoff rigidities from CALS7k.2 and CALS3k.3. Therefore, if the user select the LSD model with the Lifton (2016) VDM/VADM or the atmospheric ${ }^{10} \mathrm{Be}$ VADM (Muscheler et al., 2005) (or another VDM database), cut-off rigidities are first derived from the $\mathrm{M} / \mathrm{M}_{0}$ ratios over the whole time span. For this calculation, the program uses equation (2) proposed by Lifton et al. (2014).

Conversely, when using the "Lal modified" model with the LSD geomagnetic framework, global $\mathrm{M} / \mathrm{M}_{0}$ values derived from CALS7k.2 and CALS3k.3 are used over the $0-7 \mathrm{ka}$ BP period, leading to a loss of information about the higher order local influences on the geomagnetic field.

\subsection{Sample thickness and erosion rate correction}

CREp corrects the depth attenuation calculating the depth normalisation factor $f_{E}$. $f_{E}$ is computed by integrating the average production over the sample thickness, using a single exponential spallation attenuation equation (Balco et al., 2008):

$f_{E}=\frac{\Lambda}{\rho \cdot E}\left[1-e^{\frac{-\rho \cdot E}{\Lambda}}\right]$

E being the sample thickness $(\mathrm{cm}), \Lambda$ the spallation attenuation length $\left(\mathrm{g} \mathrm{cm}^{-2}\right), \rho\left(\mathrm{g} \mathrm{cm}^{-3}\right)$ the density. $\rho$ and $E$ must be assigned by the user. Although $\Lambda$ theoretically varies with latitude and altitude (e.g. Marrero et al., 2016), CREp uses a default constant value of $160 \mathrm{~g} \mathrm{~cm}^{-2}$ (Gosse and Phillips, 2001) (Table 1). Given the limited range of these $\Lambda$ variations, this simplification is certainly not a source of inaccuracy in the present case. It is also important to note that, in the current version of CREp, this thickness correction does not account for deep muogenic production (although muons are considered at the surface). In its present version, CREp is thus only suited to compute surface exposure ages of samples having a reasonable thickness (typically less than $20 \mathrm{~cm}$ thick). An additional muonic correction will be included in a future version of the calculator

If the erosion of the sample is known and included by the user, CREp also corrects for its effect by using the classical Equation (6) published in (Lal, 1991), i.e. assuming erosion has been constant over the exposure time.

\subsection{Shielding and topographic correction}

Several tools, such as the first CRONUS calculator (Balco et al., 2008) (http://hess.ess.washington.edu/) offer simple and efficient means for computing the shielding and topographic correction. Thus, CREp does not include a tool to compute shielding factors (see Dunne et al., 1999 for details). It is necessary to enter this previously computed correction in the input data.

\subsection{Production rates}

The CREp program proposes different possibilities to select the SLHL production rate involved in the computation of exposure ages. Users may either choose i) a worldwide mean production rate, ii) a regional value, iii) a single local value, which can be chosen among the existing dataset or imported by the user, or iv) any average from several calibration sites selected by the user (Fig. 1).

All the production rates proposed in CREp are derived from the most complete database of published calibration sites (ICE-D, see Sections 3 And 4 below).

\section{The ICE-D production rate calibration database}

The CREp program makes use of an online database of production rate calibration data (http://calibration.ice-d.org) that we have developed as part of the ICE-D (Informal Cosmogenic-nuclide Exposure-age Database) project at http://ice-d.org. Fig. 2 is a map displaying the global distribution of the ${ }^{3} \mathrm{He}$ and ${ }^{10} \mathrm{Be}$ calibration sites of the literature that are included in ICE-D.

\subsection{Motivation for creating ICE-D}

Existing compilations of production rate calibration data (Balco et al., 2008; Borchers et al., 2016; Delunel et al., 2016; Heyman, 2014) have been distributed as spreadsheet files, typically as supplementary files to published journal articles. These files are (i) incomplete, because they were developed at different moment: each one lacks some of the published production rate calibration data that were available at the time it was published; (ii) inconsistent and in most cases known to contain errors and/or omissions; and (iii) in general, limited to only the minimal set of numeric data necessary to compute an apparent production rate from each nuclide concentration measurement. Also, in some cases they are technically not available without a journal subscription, although this is not a significant obstacle for most researchers.

The current situation raises various issues. First, researchers interested in working with a comprehensive and up-to-date set of production rate calibration data must compile it themselves from a variety of sources; this requires duplicative and redundant effort, and also makes it very likely that compilations maintained by 


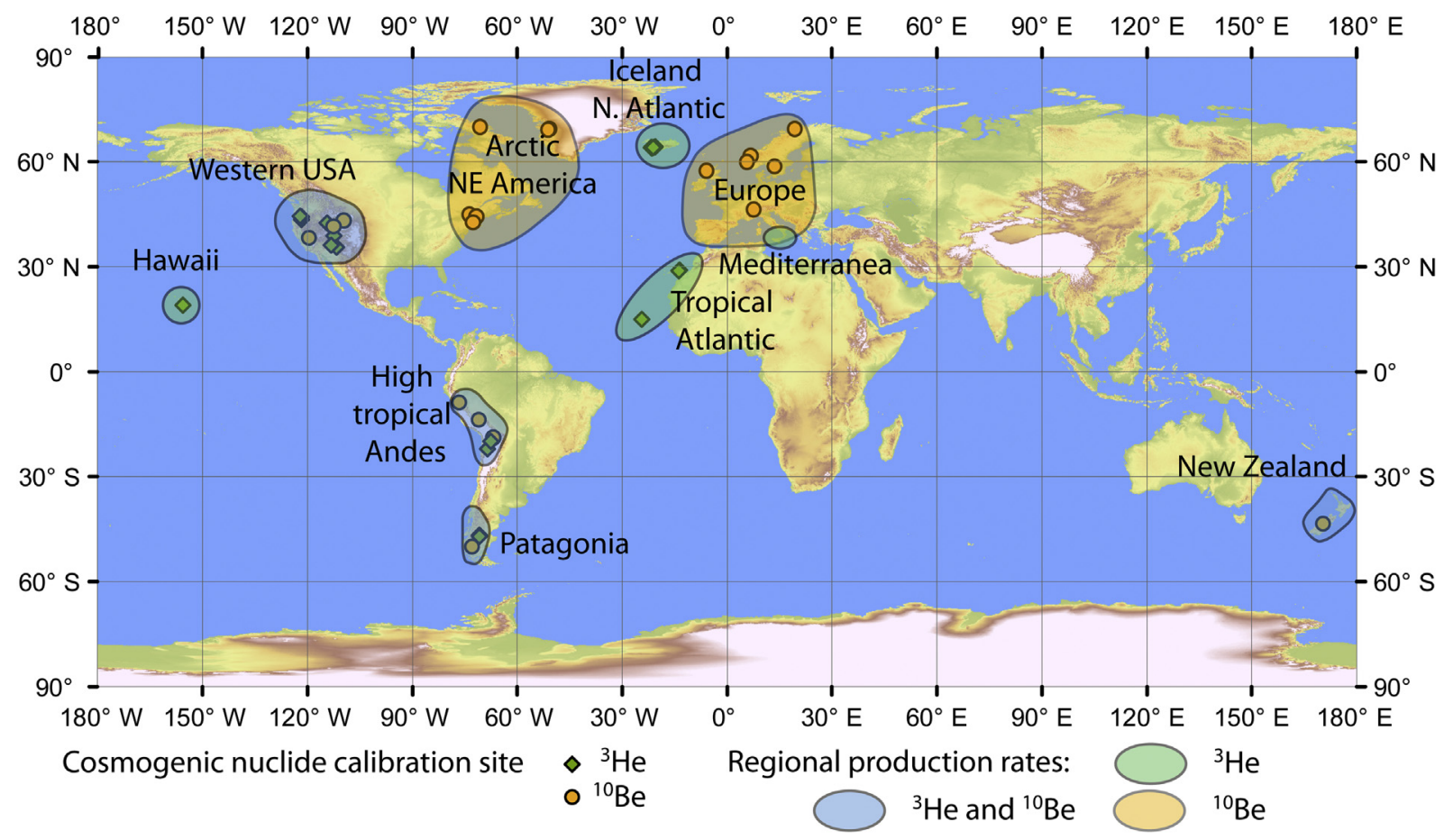

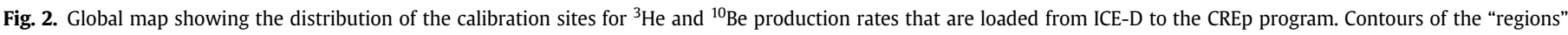
defined in the present version of CREp are also drawn.

different researchers are mutually inconsistent. Second, these compilations do not include supplementary information, such as field photos or ancillary geochemical data, that would help researchers evaluate the quality or reliability of various data sets. Third, it is difficult for researchers to share efforts in this regard because each one maintains a spreadsheet that is formatted to suit their preferred calculation methods or code.

The aim of the ICE-D database project is to improve this existing situation by providing a single online database of all known production rate calibration data that has the following properties. First, it should be updated often and checked for accuracy. Second, it should contain only direct field observations or laboratory measurements, excluding any interpreted or model-dependent parameters whose values might not be generally agreed upon, or that might be calculated in a different way in future. Third, it should be able to store not only the minimal numerical data needed to compute an apparent production rate from a measured nuclide concentration, but also ancillary information such as photos or field notes that would help researchers to evaluate site suitability and data quality. Fourth, it should follow a client-server model such that the raw data are maintained by a single server that can supply it to a wide variety of clients. Our overall goal is that any interpretive calculations related to cosmogenic-nuclide production rate calculations will always be able to address the current state of the database, rather than addressing static, locally stored data sets that might be obsolete or mutually inconsistent. Furthermore, additions, corrections, or improvements to the existing data set can simply be added to one database rather than incurring redundant efforts by many researchers. Here we describe our strategy for accomplishing these goals.

\subsection{ICE-D is designed to be accurate and complete}

To achieve this goal, we have incorporated data from all published production rate calibration studies for ${ }^{10} \mathrm{Be}$ and ${ }^{3} \mathrm{He}$ that are known to us at present, with the exception of few studies that have been shown to be affected by significant flaws or inaccuracies (Kubik et al., 1998; Kurz et al., 1990). We relied on the original publications as well as any subsequent, published or unpublished, re-evaluation or re-measurement of observations in the original paper. For example, various researchers involved in the CRONUSEarth or CRONUS-EU projects, as part of those projects, reevaluated or re-measured samples from previously described calibration sites (e.g. Phillips et al., 2016). In some cases, we corrected errors known to exist in original publications, such as few standardization issues for ${ }^{10} \mathrm{Be}$ (Balco et al., 2008; Nishiizumi et al., 2007). When necessary, we also included radiogenic ${ }^{4} \mathrm{He}$ corrections in the case of ${ }^{3} \mathrm{He}$ (Blard and Farley, 2008). In other cases, we added supporting data, documentation, or photographs that were not published, but were available to us via other sources. In some cases we have recalculated independent age constraints that were based on out-of-date radiocarbon calibrations, using IntCal13 (Reimer et al., 2013). It is certainly possible that errors or omissions that we are not aware of remain in the database. In order to facilitate locating and correcting these errors, we provide through the ICE-D project a web interface, separate and distinct from the CREp online calculator, that is indexed geographically and by publication. This interface (http://calibration.ice-d.org) allows direct examination of all data in the database. Thus, even though, as discussed above, some elements of the data set are not traceable to publications, all elements can be publicly examined for purposes of quality control. Any errors or omissions should be brought to our attention (specifically, that of authors G. Balco and P.-H. Blard) so that they can be corrected. If the data of any future calibration study are not included in ICE-D, this omission should be mentioned to these scientists (blard@crpg.cnrs-nancy.fr, balcs@bgc.org).

\subsection{Direct observations only}

We aim to record only direct observations and measurements in 


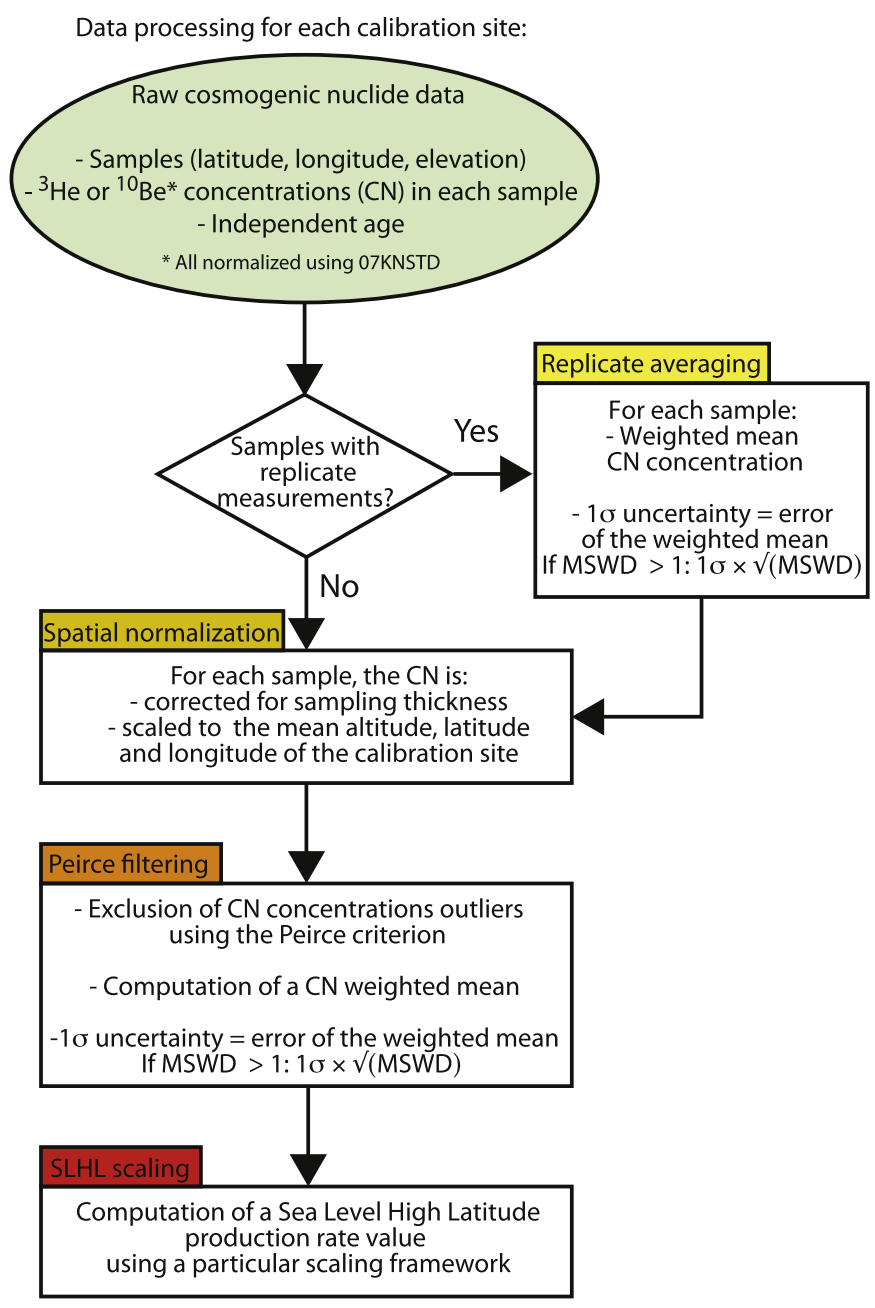

Fig. 3. Flow chart showing the raw data processing to compute a SLHL production rate from each calibration site.

the database itself. Interpretive or model-dependent decisions or assumptions - for example, which samples to include in a calibration data set, or how best to compute snow cover corrections are not hard-coded into the database. These should instead be incorporated into client software (such as CREp) that uses the database. For example, we have not renormalized ${ }^{10} \mathrm{Be}$ measurements to a common standard, but instead have recorded concentrations as they were originally measured along with information about the standardization that was used. Another example relates to thickness and shielding corrections. Where source papers reported correction factors that combined information about, for example, sample thickness, geometric shielding, and/or cover by snow or vegetation, we have decomposed these into separate observations. These are direct observations, in contrast to model-dependent attenuation factors, which might be recalculated in a different way in future. On the other hand, an example of an area where we were not able to strictly achieve this goal relates to topographic/geometric shielding factors. Although we recorded direct measurements of surface and horizon geometry (e.g., azimuth/elevation pairs recorded in the field) where they were available to us, this information is not available, and likely not recoverable, for the majority of published data. Thus, in most cases we have reported a derived topographic/geometric shielding factor rather than direct geometric observations. Fortunately, this correction is significantly less than $2 \%$ for the majority of the calibration samples and thus does not represent a significant source of inaccuracy/uncertainty.

\subsection{Ancillary information}

When possible, we incorporated field and analytical information in excess of the minimum set of numerical data needed to compute an apparent production rate from a nuclide concentration measurement. This includes information such as photographs, lithological information, field notes, dates of sample collection, names of researchers responsible for sample collection or analysis and other information or background data that could potentially be useful in data evaluation or scaling scheme development. In most such cases, we had this information because one of us was directly involved in sample collection or analysis; in some other cases, this information could be gleaned from publications. Thus, although this aspect of the database is incomplete at present, it is designed to incorporate a wide array of background information ancillary to the actual published data but potentially useful for future production rate calibration research.

\subsection{Client-server architecture}

Our aim here is to make the information in the database widely available to any software that aims to do calculations related to production rate calibration or surface exposure dating. To achieve this, we use an industry-standard model for client-server database design that is commonly used in website back ends and data management systems, and is interoperable with nearly all currently

Table 3

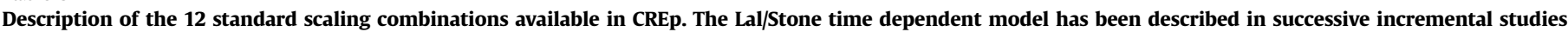

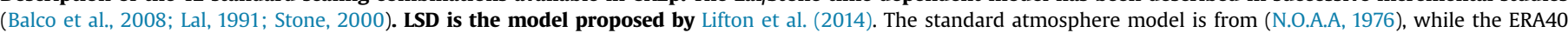

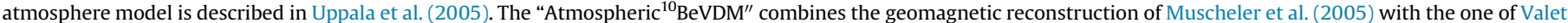
et al. (2005). The VDM called "LSD framework" is described in Lifton et al. (2014) while the "Lifton 2016 VDM" is described in Lifton (2016).

\begin{tabular}{|c|c|c|c|}
\hline Scaling shortname & Scaling scheme & Atmosphere model & Geomagnetic database \\
\hline LAL-STD-Musch & \multirow[t]{6}{*}{ Lal/Stone time dependent } & \multirow[t]{3}{*}{ Standard } & Atmospheric10Be VDM \\
\hline LAL-STD-Lift & & & Lifton 2016 VDM \\
\hline LAL-STD-L & & & LSD framework \\
\hline LAL-ERA-Musch & & \multirow[t]{3}{*}{ ERA40 } & Atmospheric10Be VDM \\
\hline LAL-ERA-Lift & & & Lifton 2016 VDM \\
\hline LAL-ERA-L & & & LSD framework \\
\hline LSD-STD-Musch & \multirow[t]{6}{*}{ LSD } & \multirow[t]{3}{*}{ Standard } & Atmospheric10Be VDM \\
\hline LSD-STD-Lift & & & Lifton 2016 VDM \\
\hline LSD-STD-L & & & LSD framework \\
\hline LSD-ERA-Musch & & \multirow[t]{3}{*}{ ERA40 } & Atmospheric10Be VDM \\
\hline LSD-ERA-Lift & & & Lifton 2016 VDM \\
\hline LSD-ERA-L & & & LSD framework \\
\hline
\end{tabular}


Global SLHL P3 dataset computed with 12 scaling procedures (n = 23). Data from (Ackert et al., 2003; Amidon and Farley, 2011; Blard et al., 2005, 2006, 2013; Cerling and Craig, 1994; Delunel et al., 2016; Dunai and Wijbrans, 2000; Fenton and Niedermann, 2014; Fenton et al., 2013; Foeken et al., 2012; Goehring et al., 2010; Licciardi et al., 1999; Poreda and Cerling, 1992).

\begin{tabular}{|c|c|c|c|c|c|c|c|c|c|c|c|c|c|c|c|c|c|}
\hline \multirow[t]{4}{*}{ Site name } & \multirow[t]{4}{*}{ Reference } & \multirow[t]{4}{*}{ Latitude } & \multirow[t]{4}{*}{ Longitude } & \multirow{4}{*}{$\begin{array}{l}\text { Altitude } \\
\overline{\text { masl }}\end{array}$} & \multirow{4}{*}{$\begin{array}{l}\text { Age } \\
\\
\mathrm{ka}\end{array}$} & \multicolumn{6}{|c|}{ Lal/Stone time dependent scaling } & \multicolumn{6}{|l|}{ LSD scaling } \\
\hline & & & & & & \multicolumn{3}{|c|}{ Standard atmosphere } & \multicolumn{3}{|c|}{ ERA40 atmosphere } & \multicolumn{3}{|c|}{ Standard atmosphere } & \multicolumn{3}{|c|}{ ERA40 atmosphere } \\
\hline & & & & & & $\begin{array}{l}\text { Muscheler } \\
\text { et al. (2005) }\end{array}$ & $\begin{array}{l}\text { Lifton } \\
\text { (2016) }\end{array}$ & LSD & $\begin{array}{l}\text { Muscheler } \\
\text { et al. (2005) }\end{array}$ & $\begin{array}{l}\text { Lifton } \\
\text { (2016) }\end{array}$ & LSD & $\begin{array}{l}\text { Muscheler } \\
\text { et al. (2005) }\end{array}$ & $\begin{array}{l}\text { Lifton } \\
\text { (2016) }\end{array}$ & LSD & $\begin{array}{l}\text { Muscheler } \\
\text { et al. (2005) }\end{array}$ & $\begin{array}{l}\text { Lifton } \\
\text { (2016) }\end{array}$ & LSD \\
\hline & & & & & & \multicolumn{12}{|l|}{ at $\mathrm{g}^{-1} \mathrm{yr}^{-1}$} \\
\hline RIOPINTU & Ackert et al., 2003 & -47.07 & -70.90 & 903 & $67.8 \pm 3.0$ & $139 \pm 7$ & $138 \pm 7$ & $136 \pm 7$ & $134 \pm 7$ & $134 \pm 7$ & $131 \pm 7$ & $138 \pm 7$ & $137 \pm 7$ & $135 \pm 7$ & $133 \pm 7$ & $133 \pm 7$ & $130 \pm 7$ \\
\hline CERRO_HIGH & Ackert et al., 2003 & -46.70 & -70.75 & 530 & $108.8 \pm 2.8$ & $138 \pm 4$ & $137 \pm 4$ & $136 \pm 4$ & $134 \pm 4$ & $134 \pm 4$ & $132 \pm 4$ & $139 \pm 4$ & $138 \pm 4$ & $136 \pm 4$ & $136 \pm 4$ & $134 \pm 4$ & $133 \pm 4$ \\
\hline CERRO_LOW & Ackert et al., 2003 & -46.61 & -70.75 & 400 & $108.8 \pm 2.8$ & $141 \pm 4$ & $140 \pm 4$ & $139 \pm 4$ & $137 \pm 4$ & $137 \pm 4$ & $135 \pm 4$ & $143 \pm 5$ & $142 \pm 4$ & $140 \pm 4$ & $139 \pm 4$ & $138 \pm 4$ & $136 \pm 4$ \\
\hline SANPEDRO & Delunel et al., 2016 & -21.93 & -68.51 & 3390 & $107.0 \pm 11.6$ & $121 \pm 13$ & $116 \pm 13$ & $110 \pm 12$ & $132 \pm 14$ & $126 \pm 14$ & $119 \pm 13$ & $117 \pm 13$ & $111 \pm 12$ & $104 \pm 11$ & $128 \pm 14$ & $122 \pm 13$ & $113 \pm 12$ \\
\hline TUNUPA & Blard et al., 2013a & -19.89 & -67.62 & 3814 & $15.2 \pm 0.5$ & $126 \pm 5$ & $122 \pm 5$ & $121 \pm 5$ & $137 \pm 5$ & $133 \pm 5$ & $132 \pm 5$ & $121 \pm 5$ & $116 \pm 4$ & $111 \pm 4$ & $133 \pm 5$ & $128 \pm 5$ & $122 \pm 5$ \\
\hline FOGO & Foeken et al., 2012 & 14.89 & -24.48 & 222 & $129.5 \pm 7.0$ & $89 \pm 5$ & $84 \pm 5$ & $81 \pm 5$ & $90 \pm 5$ & $85 \pm 5$ & $81 \pm 5$ & $101 \pm 6$ & $95 \pm 6$ & $91 \pm 6$ & $102 \pm 6$ & $97 \pm 6$ & $92 \pm 6$ \\
\hline KAUNA & Blard et al., 2006 & 19.06 & -155.55 & 60 & $8.3 \pm 0.1$ & $151 \pm 10$ & $145 \pm 10$ & $136 \pm 9$ & $153 \pm 10$ & $147 \pm 10$ & $138 \pm 9$ & $180 \pm 12$ & $168 \pm 11$ & $160 \pm 11$ & $183 \pm 12$ & $171 \pm 11$ & $164 \pm 11$ \\
\hline ATALAYA & $\begin{array}{l}\text { Dunai and } \\
\text { Wijbrans, } 2000\end{array}$ & 28.92 & -13.83 & 35 & $281.1 \pm 9.5$ & $102 \pm 4$ & $98 \pm 4$ & $97 \pm 4$ & $106 \pm 4$ & $102 \pm 4$ & $100 \pm 4$ & $122 \pm 5$ & $114 \pm 4$ & $112 \pm 4$ & $126 \pm 5$ & $118 \pm 5$ & $116 \pm 4$ \\
\hline TAHICHE & $\begin{array}{l}\text { Dunai and } \\
\text { Wijbrans, } 2000\end{array}$ & 29.01 & -13.54 & 197 & $152.1 \pm 13.0$ & $102 \pm 9$ & $99 \pm 9$ & $96 \pm 9$ & $105 \pm 9$ & $102 \pm 9$ & $99 \pm 9$ & $120 \pm 11$ & $114 \pm 10$ & $110 \pm 10$ & $124 \pm 11$ & $118 \pm 10$ & $115 \pm 10$ \\
\hline SP & $\begin{array}{l}\text { Fenton et al., 2013; } \\
\text { Fenton and } \\
\text { Niedermann, 2014; } \\
\text { Fenton et al., 2013; } \\
\text { Fenton and } \\
\text { Niedermann, } 2014\end{array}$ & 35.60 & -111.63 & 1831 & $72.0 \pm 2.0$ & $106 \pm 5$ & $105 \pm 5$ & $99 \pm 4$ & $112 \pm 5$ & $110 \pm 5$ & $104 \pm 5$ & $107 \pm 5$ & $105 \pm 5$ & $98 \pm 4$ & $113 \pm 5$ & $112 \pm 5$ & $103 \pm 5$ \\
\hline LOWBARTEN & $\begin{array}{l}\text { Fenton et al., 2013; } \\
\text { Fenton and } \\
\text { Niedermann, 2014; } \\
\text { Fenton et al., 2013; } \\
\text { Fenton and } \\
\text { Niedermann, } 2014\end{array}$ & 36.23 & -113.23 & 1226 & $123.0 \pm 6.0$ & $94 \pm 5$ & $92 \pm 5$ & $89 \pm 5$ & $98 \pm 6$ & $95 \pm 6$ & $92 \pm 5$ & $97 \pm 6$ & $94 \pm 6$ & $91 \pm 5$ & $102 \pm 6$ & $99 \pm 6$ & $95 \pm 6$ \\
\hline UPBARTEN & $\begin{array}{l}\text { Fenton et al., 2013; } \\
\text { Fenton and } \\
\text { Niedermann, 2014; } \\
\text { Fenton et al., 2013; } \\
\text { Fenton and } \\
\text { Niedermann, } 2014\end{array}$ & 36.24 & -113.19 & 1766 & $97.0 \pm 5.0$ & $107 \pm 6$ & $105 \pm 6$ & $100 \pm 6$ & $113 \pm 7$ & $110 \pm 6$ & $105 \pm 6$ & $108 \pm 6$ & $105 \pm 6$ & $99 \pm 6$ & $114 \pm 7$ & $111 \pm 7$ & $105 \pm 6$ \\
\hline NAVE & $\begin{array}{l}\text { Blard et al., 2005; } \\
\text { Blard et al., } 2006\end{array}$ & 37.85 & 14.84 & 820 & $33.1 \pm 2.0$ & $111 \pm 12$ & $109 \pm 12$ & $106 \pm 12$ & $114 \pm 12$ & $111 \pm 12$ & $109 \pm 12$ & $118 \pm 13$ & $115 \pm 13$ & $112 \pm 12$ & $121 \pm 13$ & $118 \pm 13$ & $114 \pm 12$ \\
\hline TABERNACLE & $\begin{array}{l}\text { Cerling and Craig, } \\
\text { 1994; Poreda and } \\
\text { Cerling, 1992; } \\
\text { Goehring et al., } \\
\text { 2010; Cerling and } \\
\text { Craig, 1994; Poreda } \\
\text { and Cerling, 1992; } \\
\text { Goehring et al., } \\
2010\end{array}$ & 38.93 & -112.52 & 1458 & $18.2 \pm 0.3$ & $116 \pm 2$ & $116 \pm 2$ & $115 \pm 2$ & $121 \pm 2$ & $121 \pm 2$ & $120 \pm 2$ & $117 \pm 2$ & $117 \pm 2$ & $115 \pm 2$ & $123 \pm 2$ & $122 \pm 2$ & $120 \pm 2$ \\
\hline SNAKE & $\begin{array}{l}\text { Amidon and Farley, } \\
2011\end{array}$ & 42.64 & -114.16 & 1039 & $18.3 \pm 0.3$ & $142 \pm 6$ & $142 \pm 6$ & $141 \pm 6$ & $147 \pm 6$ & $146 \pm 6$ & $145 \pm 6$ & $145 \pm 6$ & $143 \pm 6$ & $143 \pm 6$ & $149 \pm 6$ & $148 \pm 6$ & $147 \pm 6$ \\
\hline BUTTE & Licciardi et al., 1999 & 43.95 & -121.38 & 1282 & $7.1 \pm 0.1$ & $115 \pm 4$ & $112 \pm 4$ & $109 \pm 3$ & $118 \pm 4$ & $115 \pm 4$ & $112 \pm 4$ & $115 \pm 4$ & $110 \pm 4$ & $109 \pm 3$ & $118 \pm 4$ & $113 \pm 4$ & $112 \pm 4$ \\
\hline BELKNAP & Licciardi et al., 1999 & 44.24 & -121.86 & 1524 & $2.8 \pm 0.1$ & $111 \pm 6$ & $105 \pm 5$ & $104 \pm 5$ & $113 \pm 6$ & $108 \pm 6$ & $107 \pm 6$ & $108 \pm 6$ & $101 \pm 5$ & $104 \pm 5$ & $111 \pm 6$ & $104 \pm 5$ & $106 \pm 6$ \\
\hline YAPOAH & $\begin{array}{l}\text { Licciardi et al., } \\
\text { 1999; Cerling and } \\
\text { Craig, } 1994\end{array}$ & 44.27 & -121.79 & 1562 & $2.5 \pm 0.4$ & $111 \pm 18$ & $105 \pm 17$ & $104 \pm 17$ & $114 \pm 18$ & $108 \pm 17$ & $107 \pm 17$ & $108 \pm 17$ & $101 \pm 16$ & $102 \pm 17$ & $111 \pm 18$ & $103 \pm 17$ & $105 \pm 17$ \\
\hline CLEARLAKE & Licciardi et al., 1999 & 44.37 & -121.99 & 941 & $2.8 \pm 0.1$ & $119 \pm 4$ & $114 \pm 4$ & $113 \pm 4$ & $122 \pm 4$ & $117 \pm 4$ & $116 \pm 4$ & $121 \pm 4$ & $114 \pm 4$ & $117 \pm 4$ & $124 \pm 4$ & $117 \pm 4$ & $120 \pm 4$ \\
\hline LEITA & Licciardi et al., 2006 & 63.98 & -21.47 & 268 & $5.2 \pm 0.1$ & $139 \pm 6$ & $139 \pm 6$ & $139 \pm 6$ & $130 \pm 5$ & $130 \pm 5$ & $130 \pm 5$ & $133 \pm 5$ & $133 \pm 5$ & $133 \pm 5$ & $125 \pm 5$ & $124 \pm 5$ & $125 \pm 5$ \\
\hline BURFELL & Licciardi et al., 2006 & 64.09 & -21.74 & 35 & $8.1 \pm 0.1$ & $137 \pm 4$ & $137 \pm 4$ & $137 \pm 4$ & $128 \pm 4$ & $128 \pm 4$ & $128 \pm 4$ & $127 \pm 4$ & $126 \pm 4$ & $127 \pm 4$ & $119 \pm 3$ & $119 \pm 3$ & $119 \pm 3$ \\
\hline DINGVA & Licciardi et al., 2006 & 64.16 & -21.04 & 124 & $10.3 \pm 0.1$ & $138 \pm 5$ & $138 \pm 5$ & $138 \pm 5$ & $129 \pm 4$ & $129 \pm 4$ & $129 \pm 4$ & $129 \pm 4$ & $129 \pm 4$ & $129 \pm 4$ & $122 \pm 4$ & $121 \pm 4$ & $121 \pm 4$ \\
\hline LAMBA & Licciardi et al., 2006 & 64.37 & -20.55 & 452 & $4.0 \pm 0.3$ & $128 \pm 10$ & $128 \pm 10$ & $128 \pm 10$ & $119 \pm 9$ & $119 \pm 9$ & $119 \pm 9$ & $122 \pm 10$ & $122 \pm 10$ & $122 \pm 10$ & $114 \pm 9$ & $114 \pm 9$ & $114 \pm 9$ \\
\hline
\end{tabular}


Table 5

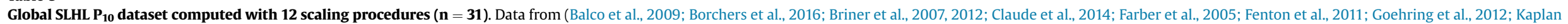
et al., 2011; Kelly et al., 2015; Lifton et al., 2015; Martin et al., 2015; Nishiizumi et al., 1989; Phillips et al., 2016; Putnam et al., 2010; Young et al., 2013).

\begin{tabular}{|c|c|c|c|c|c|c|c|c|c|c|c|c|c|c|c|c|c|}
\hline \multirow[t]{4}{*}{ Site name } & \multirow[t]{4}{*}{ Reference } & \multirow[t]{4}{*}{ Latitude } & \multirow[t]{4}{*}{ Longitude } & \multirow[t]{3}{*}{ Altitude } & \multirow[t]{3}{*}{ Age } & \multicolumn{6}{|c|}{ Lal/Stone time dependent scaling } & \multicolumn{6}{|l|}{ LSD scaling } \\
\hline & & & & & & \multicolumn{3}{|c|}{ Standard atmosphere } & \multicolumn{3}{|c|}{ ERA40 atmosphere } & \multicolumn{3}{|c|}{ Standard atmosphere } & \multicolumn{3}{|c|}{ ERA40 atmosphere } \\
\hline & & & & & & $\begin{array}{l}\text { Muscheler } \\
\text { et al. (2005) }\end{array}$ & Lifton (2016) & LSD & $\begin{array}{l}\text { Muscheler } \\
\text { et al. (2005) }\end{array}$ & Lifton (2016) & LSD & $\begin{array}{l}\text { Muscheler } \\
\text { et al. (2005) }\end{array}$ & Lifton (2016) & LSD & $\begin{array}{l}\text { Muscheler } \\
\text { et al. (2005) }\end{array}$ & Lifton (2016) & LSD \\
\hline & & & & masl & years & at $\mathrm{g}^{-1} \mathrm{yr}^{-1}$ & & & & & & & & & & & \\
\hline BANDERA & Kaplan et al., 2011 & -50.20 & -72.70 & 220 & $12825 \pm 480$ & $4.25 \pm 0.17$ & $4.24 \pm 0.16$ & $4.23 \pm 0.16$ & $4.05 \pm 0.16$ & $4.03 \pm 0.16$ & $4.02 \pm 0.16$ & $4.29 \pm 0.17$ & $4.27 \pm 0.17$ & $4.26 \pm 0.17$ & $4.08 \pm 0.16$ & $4.06 \pm 0.16$ & $4.06 \pm 0.16$ \\
\hline BST & Putnam et al., 2010 & -44.04 & 170.12 & 841.8 & $18,200 \pm 200$ & $4.06 \pm 0.06$ & $4.05 \pm 0.06$ & $4.02 \pm 0.06$ & $4.02 \pm 0.06$ & $4.01 \pm 0.06$ & $3.99 \pm 0.06$ & $4.20 \pm 0.06$ & $4.16 \pm 0.06$ & $4.06 \pm 0.06$ & $4.15 \pm 0.06$ & $4.12 \pm 0.06$ & $4.02 \pm 0.06$ \\
\hline MACAULAY & Putnam et al., 2010 & -43.58 & 170.61 & 1028 & $9634 \pm 50$ & $4.12 \pm 0.04$ & $4.07 \pm 0.04$ & $3.98 \pm 0.04$ & $4.08 \pm 0.04$ & $4.03 \pm 0.04$ & $3.95 \pm 0.04$ & $4.25 \pm 0.04$ & $4.15 \pm 0.04$ & $3.87 \pm 0.04$ & $4.21 \pm 0.04$ & $4.13 \pm 0.04$ & $3.86 \pm 0.04$ \\
\hline & Martin et al., 2015 & -18.91 & -66.75 & 3889 & $16,010 \pm 640$ & $3.83 \pm 0.16$ & $3.75 \pm 0.16$ & $3.70 \pm 0.15$ & $4.16 \pm 0.17$ & $4.08 \pm 0.17$ & $4.03 \pm 0.17$ & $4.06 \pm 0.17$ & $3.89 \pm 0.16$ & $3.74 \pm 0.15$ & $4.44 \pm 0.18$ & $4.29 \pm 0.18$ & $4.10 \pm 0.17$ \\
\hline HUANCANE2A & $\begin{array}{l}\text { Kelly et al., 2015; } \\
\text { Phillips et al., } 2016\end{array}$ & -13.95 & -70.89 & 4858 & $12,320 \pm 110$ & $3.91 \pm 0.18$ & $3.78 \pm 0.17$ & $3.66 \pm 0.17$ & $4.28 \pm 0.20$ & $4.14 \pm 0.19$ & $4.01 \pm 0.18$ & $3.98 \pm 0.18$ & $3.76 \pm 0.17$ & $3.49 \pm 0.16$ & $4.39 \pm 0.20$ & $4.15 \pm 0.19$ & $3.86 \pm 0.18$ \\
\hline BREQUE & Farber et al., 2005 & -9.65 & -77.36 & 4045 & $13,000 \pm 100$ & $4.18 \pm 0.12$ & $4.05 \pm 0.11$ & $3.94 \pm 0.11$ & $4.51 \pm 0.13$ & $4.37 \pm 0.12$ & $4.25 \pm 0.12$ & $4.30 \pm 0.12$ & $4.04 \pm 0.11$ & $3.80 \pm 0.11$ & $4.67 \pm 0.13$ & $4.39 \pm 0.12$ & $4.13 \pm 0.12$ \\
\hline BABOON & Borchers et al., 2016 & 37.17 & -118.62 & 3385 & $13,300 \pm 250$ & $3.80 \pm 0.15$ & $3.72 \pm 0.14$ & $3.66 \pm 0.14$ & $4.02 \pm 0.15$ & $3.93 \pm 0.15$ & $3.87 \pm 0.15$ & $3.75 \pm 0.14$ & $3.64 \pm 0.14$ & $3.51 \pm 0.13$ & $3.98 \pm 0.15$ & $3.90 \pm 0.15$ & $3.76 \pm 0.14$ \\
\hline STARR & $\begin{array}{l}\text { Borchers et al., 2016; } \\
\text { Nishiizumi et al., 1989; } \\
\text { Balco et al., 2008; } \\
\text { Borchers et al., 2016; } \\
\text { Nishiizumi et al., 1989; } \\
\text { Balco et al., } 2008\end{array}$ & 37.42 & -118.77 & 3556 & $15,750 \pm 500$ & $4.13 \pm 0.32$ & $4.07 \pm 0.31$ & $4.03 \pm 0.31$ & $4.37 \pm 0.34$ & $4.30 \pm 0.33$ & $4.26 \pm 0.33$ & $4.01 \pm 0.31$ & $3.95 \pm 0.30$ & $3.84 \pm 0.29$ & $4.26 \pm 0.33$ & $4.20 \pm 0.32$ & $4.09 \pm 0.31$ \\
\hline GREENS & $\begin{array}{l}\text { Borchers et al., 2016; } \\
\text { Nishiizumi et al., 1989; } \\
\text { Balco et al., 2008; } \\
\text { Borchers et al., 2016; } \\
\text { Nishiizumi tel.l, } 989 \text {; } \\
\text { Balco et al., 2008 }\end{array}$ & 37.98 & -119.30 & 3175 & $15,750 \pm 500$ & $3.72 \pm 0.41$ & $3.66 \pm 0.40$ & $3.63 \pm 0.40$ & $3.92 \pm 0.43$ & $3.86 \pm 0.42$ & $3.82 \pm 0.42$ & $3.64 \pm 0.40$ & $3.59 \pm 0.39$ & $3.51 \pm 0.38$ & $3.85 \pm 0.42$ & $3.80 \pm 0.41$ & $3.71 \pm 0.40$ \\
\hline TWINS & $\begin{array}{l}\text { Borchers et al., 2016; } \\
\text { Nishiizumi et al., 1989; } \\
\text { Balco et al., 2008; } \\
\text { Borchers et al., 2016; } \\
\text { Nishiizumi et al., 1989; } \\
\text { Balco et al., } 2008\end{array}$ & 38.87 & -120.20 & 2371 & $15,750 \pm 500$ & $3.88 \pm 0.29$ & $3.83 \pm 0.28$ & $3.79 \pm 0.28$ & $4.05 \pm 0.30$ & $4.00 \pm 0.30$ & $3.96 \pm 0.29$ & $3.91 \pm 0.29$ & $3.86 \pm 0.29$ & $3.79 \pm 0.28$ & $4.10 \pm 0.30$ & $4.05 \pm 0.30$ & $3.97 \pm 0.29$ \\
\hline PPT & $\begin{array}{l}\text { Lifton et al., 2015; } \\
\text { Borchers et al., } 2016\end{array}$ & 41.26 & -112.48 & 1603 & $18,300 \pm 300$ & $4.10 \pm 0.07$ & $4.08 \pm 0.07$ & $4.05 \pm 0.07$ & $4.27 \pm 0.07$ & $4.24 \pm 0.07$ & $4.21 \pm 0.07$ & $4.18 \pm 0.07$ & $4.15 \pm 0.07$ & $4.10 \pm 0.07$ & $4.36 \pm 0.07$ & $4.32 \pm 0.07$ & $4.28 \pm 0.07$ \\
\hline GLASH & Balco et al., 2009 & 43.01 & -72.33 & 182 & $15,145 \pm 300$ & $4.05 \pm 0.11$ & $4.03 \pm 0.11$ & $4.01 \pm 0.10$ & $4.13 \pm 0.11$ & $4.10 \pm 0.11$ & $4.08 \pm 0.11$ & $4.33 \pm 0.11$ & $4.27 \pm 0.11$ & $4.25 \pm 0.11$ & $4.41 \pm 0.12$ & $4.35 \pm 0.11$ & $4.33 \pm 0.11$ \\
\hline TITCOMB & $\begin{array}{l}\text { Gosse et al., 1995; } \\
\text { Borchers et al., 2016; } \\
\text { Balco et al., 2008; Gosse } \\
\text { et al., 1995; Borchers } \\
\text { et al., 2016; Balco et al., } \\
2008\end{array}$ & 43.12 & -109.64 & 3231 & $12,100 \pm 700$ & $4.86 \pm 0.29$ & $4.79 \pm 0.28$ & $4.72 \pm 0.28$ & $5.06 \pm 0.30$ & $4.99 \pm 0.29$ & $4.92 \pm 0.29$ & $4.48 \pm 0.27$ & $4.40 \pm 0.26$ & $4.33 \pm 0.26$ & $4.69 \pm 0.28$ & $4.60 \pm 0.27$ & $4.53 \pm 0.27$ \\
\hline SLAM & Balco et al., 2009 & 44.29 & -71.76 & 357 & $13,900 \pm 250$ & $4.04 \pm 0.12$ & $4.01 \pm 0.12$ & $3.99 \pm 0.12$ & $4.08 \pm 0.12$ & $4.05 \pm 0.12$ & $4.03 \pm 0.12$ & $4.26 \pm 0.12$ & $4.20 \pm 0.12$ & $4.18 \pm 0.12$ & $4.30 \pm 0.13$ & $4.24 \pm 0.12$ & $4.23 \pm 0.12$ \\
\hline BEECH & $\begin{array}{l}\text { Balco et al., 2009; } \\
\text { Borchers et al., } 2016\end{array}$ & 44.31 & -71.58 & 412 & $13,900 \pm 250$ & $4.18 \pm 0.09$ & $4.15 \pm 0.09$ & $4.12 \pm 0.09$ & $4.22 \pm 0.09$ & $4.19 \pm 0.09$ & $4.16 \pm 0.09$ & $4.38 \pm 0.10$ & $4.32 \pm 0.09$ & $4.30 \pm 0.09$ & $4.42 \pm 0.10$ & $4.36 \pm 0.09$ & $4.34 \pm 0.09$ \\
\hline COBBLE & Balco et al., 2009 & 44.85 & -73.59 & 234 & $13,180 \pm 130$ & $3.72 \pm 0.11$ & $3.70 \pm 0.11$ & $3.68 \pm 0.11$ & $3.78 \pm 0.12$ & $3.75 \pm 0.11$ & $3.73 \pm 0.11$ & $3.91 \pm 0.12$ & $3.85 \pm 0.12$ & $3.84 \pm 0.12$ & $3.96 \pm 0.12$ & $3.92 \pm 0.12$ & $3.90 \pm 0.12$ \\
\hline CHI & Claude et al., 2014 & 46.42 & 8.85 & 782 & $13,328 \pm 113$ & $4.02 \pm 0.10$ & $3.99 \pm 0.10$ & $3.97 \pm 0.10$ & $4.11 \pm 0.10$ & $4.08 \pm 0.10$ & $4.06 \pm 0.10$ & $4.10 \pm 0.10$ & $4.06 \pm 0.10$ & $4.05 \pm 0.10$ & $4.20 \pm 0.10$ & $4.16 \pm 0.10$ & $4.15 \pm 0.10$ \\
\hline KYPASS & Borchers et al., 2016 & 57.22 & -5.72 & 310 & $11,700 \pm 300$ & $4.58 \pm 0.26$ & $4.58 \pm 0.26$ & $4.58 \pm 0.26$ & $4.48 \pm 0.26$ & $4.48 \pm 0.26$ & $4.48 \pm 0.26$ & $4.46 \pm 0.26$ & $4.45 \pm 0.25$ & $4.44 \pm 0.25$ & $4.36 \pm 0.25$ & $4.35 \pm 0.25$ & $4.36 \pm 0.25$ \\
\hline FEAR & Borchers et al., 2016 & 57.24 & -5.97 & 336 & $11,700 \pm 300$ & $4.35 \pm 0.13$ & $4.35 \pm 0.13$ & $4.35 \pm 0.13$ & $4.25 \pm 0.13$ & $4.25 \pm 0.13$ & $4.25 \pm 0.13$ & $4.24 \pm 0.12$ & $4.23 \pm 0.12$ & $4.22 \pm 0.12$ & $4.14 \pm 0.12$ & $4.13 \pm 0.12$ & $4.13 \pm 0.12$ \\
\hline ARR & Borchers et al., 2016 & 57.42 & -5.65 & 135 & $11,700 \pm 300$ & $4.38 \pm 0.12$ & $4.38 \pm 0.12$ & $4.38 \pm 0.12$ & $4.29 \pm 0.11$ & $4.29 \pm 0.11$ & $4.29 \pm 0.11$ & $4.21 \pm 0.11$ & $4.20 \pm 0.11$ & $4.21 \pm 0.11$ & $4.13 \pm 0.11$ & $4.12 \pm 0.11$ & $4.12 \pm 0.11$ \\
\hline MCD & Borchers et al., 2016 & 57.49 & -5.45 & 515 & $11,700 \pm 300$ & $4.33 \pm 0.12$ & $4.33 \pm 0.12$ & $4.33 \pm 0.12$ & $4.22 \pm 0.11$ & $4.21 \pm 0.11$ & $4.21 \pm 0.11$ & $4.23 \pm 0.11$ & $4.22 \pm 0.11$ & $4.21 \pm 0.11$ & $4.12 \pm 0.11$ & $4.11 \pm 0.11$ & $4.11 \pm 0.11$ \\
\hline BILL3 & Stroeven et al., 2015 & 58.51 & 13.63 & 105 & $11,061 \pm 200$ & $4.31 \pm 0.31$ & $4.31 \pm 0.31$ & $4.31 \pm 0.31$ & $4.27 \pm 0.30$ & $4.28 \pm 0.30$ & $4.28 \pm 0.30$ & $4.10 \pm 0.29$ & $4.10 \pm 0.29$ & $4.09 \pm 0.29$ & $4.07 \pm 0.29$ & $4.07 \pm 0.29$ & $4.06 \pm 0.29$ \\
\hline BILL6 & Stroeven et al., 2015 & 58.51 & 13.68 & 120 & $11,477 \pm 110$ & $4.38 \pm 0.18$ & $4.38 \pm 0.18$ & $4.39 \pm 0.18$ & $4.34 \pm 0.18$ & $4.35 \pm 0.18$ & $4.35 \pm 0.18$ & $4.18 \pm 0.17$ & $4.16 \pm 0.17$ & $4.16 \pm 0.17$ & $4.15 \pm 0.17$ & $4.14 \pm 0.17$ & $4.14 \pm 0.17$ \\
\hline BILL1 & Stroeven et al., 2015 & 58.53 & 13.76 & 95 & $10,680 \pm 351$ & $4.20 \pm 0.30$ & $4.20 \pm 0.30$ & $4.20 \pm 0.30$ & $4.17 \pm 0.30$ & $4.17 \pm 0.30$ & $4.17 \pm 0.30$ & $3.99 \pm 0.29$ & $3.98 \pm 0.28$ & $3.97 \pm 0.28$ & $3.96 \pm 0.28$ & $3.95 \pm 0.28$ & $3.95 \pm 0.28$ \\
\hline HALSNOY & Goehring et al., 2012 & 59.80 & 5.80 & 84 & $11,590 \pm 100$ & $4.53 \pm 0.08$ & $4.53 \pm 0.08$ & $4.54 \pm 0.08$ & $4.45 \pm 0.08$ & $4.46 \pm 0.08$ & $4.46 \pm 0.08$ & $4.10 \pm 0.10$ & $4.27 \pm 0.08$ & $4.27 \pm 0.08$ & $4.21 \pm 0.08$ & $4.21 \pm 0.07$ & $4.20 \pm 0.07$ \\
\hline OLDEDALEN & Goehring et al., 2012 & 61.67 & 6.81 & 135 & $6010 \pm 110$ & $4.36 \pm 0.14$ & $4.36 \pm 0.14$ & $4.36 \pm 0.14$ & $4.25 \pm 0.14$ & $4.25 \pm 0.14$ & $4.25 \pm 0.14$ & $4.14 \pm 0.14$ & $4.12 \pm 0.14$ & $4.11 \pm 0.14$ & $4.04 \pm 0.13$ & $4.03 \pm 0.13$ & $4.03 \pm 0.13$ \\
\hline GROTLANDSURA & Fenton et al., 2011 & 68.91 & 17.53 & 53 & $11,424 \pm 108$ & $3.66 \pm 0.10$ & $3.66 \pm 0.10$ & $3.66 \pm 0.10$ & $3.50 \pm 0.10$ & $3.50 \pm 0.10$ & $3.50 \pm 0.10$ & $3.40 \pm 0.10$ & $3.40 \pm 0.10$ & $3.40 \pm 0.10$ & $3.27 \pm 0.09$ & $3.27 \pm 0.09$ & $3.27 \pm 0.09$ \\
\hline TASIUSSAQ & $\begin{array}{l}\text { Briner et al., 2012; } \\
\text { Young et al., 2013 }\end{array}$ & 69.15 & -51.04 & 143 & $8177 \pm 360$ & $4.34 \pm 0.19$ & $4.34 \pm 0.19$ & $4.34 \pm 0.19$ & $4.13 \pm 0.18$ & $4.13 \pm 0.18$ & $4.13 \pm 0.18$ & $4.06 \pm 0.18$ & $4.05 \pm 0.18$ & $4.06 \pm 0.18$ & $3.90 \pm 0.17$ & $3.90 \pm 0.17$ & $3.90 \pm 0.17$ \\
\hline RUSSENES & Fenton et al., 2011 & 69.21 & 19.47 & 105 & $10,942 \pm 77$ & $4.04 \pm 0.14$ & $4.04 \pm 0.14$ & $4.04 \pm 0.14$ & $3.85 \pm 0.13$ & $3.85 \pm 0.13$ & $3.85 \pm 0.13$ & $3.78 \pm 0.13$ & $3.78 \pm 0.13$ & $3.77 \pm 0.13$ & $3.63 \pm 0.12$ & $3.63 \pm 0.12$ & $3.63 \pm 0.12$ \\
\hline MARRAIT & Young et al., 2013 & 69.28 & -50.76 & 350 & $9175 \pm 45$ & $4.32 \pm 0.05$ & $4.32 \pm 0.05$ & $4.32 \pm 0.05$ & $4.11 \pm 0.05$ & $4.11 \pm 0.05$ & $4.11 \pm 0.05$ & $4.12 \pm 0.05$ & $4.11 \pm 0.05$ & $4.12 \pm 0.05$ & $3.92 \pm 0.05$ & $3.92 \pm 0.05$ & $3.92 \pm 0.05$ \\
\hline CLYDE & $\begin{array}{l}\text { Briner et al., 2007; } \\
\text { Balco et al., 2009; } \\
\text { Young et al., } 2013\end{array}$ & 69.83 & -70.50 & 67 & $8193 \pm 50$ & $4.30 \pm 0.10$ & $4.30 \pm 0.10$ & $4.30 \pm 0.10$ & $4.22 \pm 0.10$ & $4.22 \pm 0.10$ & $4.22 \pm 0.10$ & $3.99 \pm 0.09$ & $3.99 \pm 0.09$ & $3.99 \pm 0.09$ & $3.92 \pm 0.09$ & $3.92 \pm 0.09$ & $3.92 \pm 0.09$ \\
\hline
\end{tabular}


available computational and data-management software. The database itself occupies a MySQL server that is currently hosted by the Google Cloud SQL service (http://cloud.google.com/sql). As this is a standard database format, however, it is not fundamentally linked to any particular hosting service, and any equivalent service could be used. The structure and organization of the database can be examined via the browsing interface at http://calibration.ice-d. org. The database is accessible (subject to reasonable security restrictions) to any client software capable of making a SQL query, which includes essentially all modern programming languages as well as commonly used desktop calculation environments such as MATLAB, Mathematica, iPython, or R. The online version of the CREp calculator as well as the web server at http://calibration.ice-d. org are two examples of such client software.

\section{Implementation of the ICE-D production rates in CREp}

The CREp website client has daily access to the content of the ICE-D calibration database to read the calibration data, so that any modification of the ICE-D database is almost instantaneously incorporated in CREp.

CREp incorporates these ${ }^{3} \mathrm{He}$ and ${ }^{10} \mathrm{Be}$ calibration data to compute local, regional and global production rates. Note that CREp only includes in its dataset the calibration sites having multiple surface samples. Additionally, CREp only includes landforms whose age is constrained by at least 2 bracketing independent ages, or that are directly dated (e.g. $\mathrm{Ar}-\mathrm{Ar}$ or $\mathrm{K}-\mathrm{Ar}$-dated lava flows, or ${ }^{14} \mathrm{C}$ from wood material in a landslide); sites having only one limiting (maximum or minimum) age are excluded. These default arbitrary choices can however be easily overcome: for this, users have to use the "Load your own calibration data" function of CREp.

\subsection{Calculation of a production rate from each calibration site}

CREp calculates a production rate from each calibration site applying the following flow chart (Fig. 3):

- Multiple aliquots averaging: If several ${ }^{3} \mathrm{He}$ or ${ }^{10} \mathrm{Be}$ laboratory measurements are reported for a single sample, an errorweighted-mean concentration and its associated error weighted uncertainty are computed from those measurements. The mean squared weighted deviation (MSWD, also known as the reduced $\chi^{2}$ ) is also calculated to test the data distribution. If MSWD $>1$, the error-weighted mean is corrected for this overdispersion by multiplying the uncertainty by $\sqrt{ }($ MSWD $)$ (Douglass et al., 2006; York, 1966).

- Spatial normalization: For each sample, the cosmogenic nuclide concentration is then corrected for sampling thickness (normalized to the surface, i.e. null thickness), topographic shielding and scaled to a mean altitude, latitude and longitude representative of each calibration site. In order to be internally consistent, this local normalization scaling uses the scaling procedure (scheme, atmosphere model, geomagnetic database) corresponding to the choice of the user. Note that the spatial range of all the ICE-D calibration samples is narrow, implying that this correction is rather limited (less than 5\%) between the different samples. This normalization is very useful for analyzing the sample distribution on each site and detecting outliers (that could be affected by inheritance or erosion).

- Outlier removal and weighted-mean computation: For each calibration site having more than 2 samples, the distribution of the sample concentrations is evaluated applying the criterion of Peirce (Peirce, 1852; Ross, 2003). This filtering is motivated to exclude samples that are affected by erosion or inheritance (or an undetected analytical bias). Then, a weighted mean concentration is computed for each calibration site, and the associated one-sigma uncertainty is the standard error of the weighted mean $\sigma_{\mathrm{w}}$. This is justified since these surface samples are similar to multiple measurements of the same physical property, i.e. the cosmogenic nuclide concentration of a supposedly synchronous geomorphological surface. Note that, in the case of sites having MSWD larger than $1, \sigma_{\mathrm{w}}$ is multiplied by $\sqrt{ }($ MSWD) to account for this overdispersion (Douglass et al., 2006; York, 1966).

The individual ${ }^{3} \mathrm{He}$ production rates (further referred as $\mathrm{P}_{3}$ ) calculated in this way range from $81 \pm 5$ to $185 \pm 12$ at g $^{-1} \mathrm{yr}^{-1}$, for global averages of $116 \pm 17$ to $123 \pm 11$ at g ${ }^{-1} \mathrm{yr}^{-1}$ (uncertainties attached to the global means are weighted standard deviations), depending on the scaling framework used (Table 4). Similarly, the ${ }^{10} \mathrm{Be}$ production rates (further referred as $\mathrm{P}_{10}$ ) range from $3.27 \pm 0.09$ to $5.06 \pm 0.30$ at $\mathrm{g}^{-1} \mathrm{yr}^{-1}$, for global averages of $3.98 \pm 0.23$ to $4.16 \pm 0.20$ at $\mathrm{g}^{-1} \mathrm{yr}^{-1}$ (uncertainty attached to the global means are weighted standard deviations), depending on the scaling framework used (Table 5).

\subsection{Arbitrary combination of any calibration sites}

CREp allows selecting any arbitrary combination of production rates from the calibration data available in ICE-D. This option is proposed for flexibility and transparency, to permit users to test different combination of production rates, and the impact of this choice on the computed exposure ages. For this, users simply have to select the sites by clicking on the displayed online map (http:// crep.crpg.cnrs-nancy.fr/\#/production-rate) (Fig. A4 in Appendix 2). If more than one site is selected, CREp computes the weighted mean of the selected production rates and the MSWD. The uncertainty associated with this selection of production rates is evaluated by taking the largest value between the error of the weighted mean and the weighted mean standard deviation. It is worth noting that this selection function does not perform any outlier exclusion since it is designed to allow the use of any arbitrary combination of calibration data.

\subsection{Calculation of regional production rates}

Several studies (e.g. Balco et al., 2009; Martin et al., 2015; Stroeven et al., 2015) proposed the use of "regionally averaged" production rate. This approach aims to overcome several caveats, such as the relative inability of scaling schemes to account for local peculiarities in certain regions (due for example from atmospheric or geomagnetic anomalies that have not been adequately modeled). Moreover, regionally averaged rates have the advantage to be statistically more robust than single local calibration sites, and may be more likely to account for unrecognized geologic/geomorphic inaccuracies (e.g. erosion, partial shielding, inaccurate dating) associated with particular sites.

CREp offers the possibility to use this approach, by selecting precomputed regional production rates yielded by the average of several local calibration sites. To obtain these average regional rates, CREp applies the following procedure: if the region has only 2 calibration sites, the weighted average of the 2 sites and its associated error are computed.

If the region includes 3 or more calibration sites, these sitespecific SLHL production rates are filtered applying the Peirce criterion (Peirce, 1852; Ross, 2003). For each regional dataset, CREp then computes the weighted mean, the weighted standard deviation as well as the error of the weighted mean. The largest of these uncertainties is then used as the error of the regional dataset.

The limits of these regions were determined by taking into 
Table 6

Regional SLHL $\mathrm{P}_{3}$ and $\mathrm{P}_{10}$ as defined in CREp computed with 12 scaling procedures.

\begin{tabular}{|c|c|c|c|c|c|c|c|c|c|c|c|c|c|}
\hline \multirow[t]{2}{*}{ Nuclide } & \multirow[t]{2}{*}{ Region } & LAL-STD-Musch ${ }^{\mathrm{a}}$ & LAL-STD-Lift & LAL-STD-L & LAL-ERA-Musch & LAL-ERA-Lift & LAL-ERA-L & LSD-STD-Musch & LSD-STD-Lift & LSD-STD-L & LSD-ERA-Musch & LSD-ERA-Lift & LSD-ERA-L \\
\hline & & \multicolumn{12}{|l|}{ at $\mathrm{g}^{-1} \mathrm{yr}^{-1}$} \\
\hline \multirow[t]{7}{*}{$3 \mathrm{He}$} & Iceland North Atlantic & $138 \pm 3$ & $138 \pm 3$ & $138 \pm 3$ & $129 \pm 2$ & $129 \pm 2$ & $129 \pm 2$ & $128 \pm 3$ & $128 \pm 3$ & $128 \pm 3$ & $121 \pm 3$ & $121 \pm 3$ & $121 \pm 3$ \\
\hline & Tropical Atlantic North Africa & $98 \pm 7$ & $94 \pm 8$ & $91 \pm 9$ & $101 \pm 9$ & $96 \pm 10$ & $94 \pm 11$ & $114 \pm 12$ & $108 \pm 10$ & $105 \pm 12$ & $118 \pm 14$ & $111 \pm 12$ & $12 \pm 108$ \\
\hline & High Tropical Andes & $125 \pm 4$ & $122 \pm 4$ & $119 \pm 6$ & $136 \pm 5$ & $132 \pm 5$ & $130 \pm 6$ & $121 \pm 4$ & $116 \pm 4$ & $110 \pm 4$ & $132 \pm 5$ & $127 \pm 5$ & $5 \pm 121$ \\
\hline & $\begin{array}{l}\text { Patagonia } \\
\text { Pata }\end{array}$ & $139 \pm 3$ & $139 \pm 3$ & $137 \pm 3$ & $135 \pm 3$ & $135 \pm 3$ & $133 \pm 3$ & $141 \pm 3$ & $139 \pm 3$ & $137 \pm 3$ & $137 \pm 3$ & $136 \pm 3$ & $3 \pm 134$ \\
\hline & Hawaii Central Pacific & $151 \pm 10$ & $145 \pm 10$ & $136 \pm 9$ & $153 \pm 10$ & $147 \pm 10$ & $138 \pm 9$ & $180 \pm 12$ & $168 \pm 11$ & $160 \pm 11$ & $183 \pm 12$ & $171 \pm 11$ & $11 \pm 164$ \\
\hline & Western USA & $113 \pm 7$ & $111 \pm 7$ & $109 \pm 8$ & $118 \pm 7$ & $115 \pm 7$ & $113 \pm 8$ & $114 \pm 6$ & $111 \pm 7$ & $109 \pm 9$ & $119 \pm 7$ & $116 \pm 8$ & $8 \pm 114$ \\
\hline & Mediterranean & $111 \pm 12$ & $109 \pm 12$ & $106 \pm 12$ & $114 \pm 12$ & $111 \pm 12$ & $109 \pm 12$ & $118 \pm 13$ & $115 \pm 13$ & $112 \pm 12$ & $121 \pm 13$ & $118 \pm 13$ & $13 \pm 114$ \\
\hline \multirow[t]{6}{*}{ 10Be } & High Tropical Andes & $4.02 \pm 0.20$ & $3.91 \pm 0.17$ & $3.81 \pm 0.16$ & $4.37 \pm 0.19$ & $4.25 \pm 0.16$ & $4.14 \pm 0.14$ & $4.16 \pm 0.17$ & $3.94 \pm 0.14$ & $3.72 \pm 0.15$ & $4.55 \pm 0.16$ & $4.31 \pm 0.12$ & $4.06 \pm 0.14$ \\
\hline & Patagonia & $4.25 \pm 0.17$ & $4.24 \pm 0.16$ & $4.23 \pm 0.16$ & $4.05 \pm 0.16$ & $4.03 \pm 0.16$ & $4.02 \pm 0.16$ & $4.29 \pm 0.17$ & $4.27 \pm 0.17$ & $4.26 \pm 0.17$ & $4.08 \pm 0.16$ & $4.06 \pm 0.16$ & $4.06 \pm 0.16$ \\
\hline & New Zealand SW Pacific & $4.10 \pm 0.04$ & $4.06 \pm 0.03$ & $3.99 \pm 0.03$ & $4.06 \pm 0.04$ & $4.02 \pm 0.03$ & $3.96 \pm 0.03$ & $4.23 \pm 0.03$ & $4.15 \pm 0.03$ & $3.93 \pm 0.12$ & $4.19 \pm 0.04$ & $4.13 \pm 0.03$ & $3.91 \pm 0.11$ \\
\hline & Western USA & $4.04 \pm 0.14$ & $4.00 \pm 0.17$ & $3.96 \pm 0.18$ & $4.21 \pm 0.13$ & $4.17 \pm 0.15$ & $4.14 \pm 0.16$ & $4.09 \pm 0.22$ & $4.04 \pm 0.24$ & $3.97 \pm 0.28$ & $4.27 \pm 0.18$ & $4.24 \pm 0.21$ & $4.17 \pm 0.24$ \\
\hline & Europe & $4.32 \pm 0.20$ & $4.31 \pm 0.21$ & $4.31 \pm 0.21$ & $4.25 \pm 0.18$ & $4.25 \pm 0.18$ & $4.25 \pm 0.19$ & $4.13 \pm 0.14$ & $4.16 \pm 0.15$ & $4.15 \pm 0.15$ & $4.11 \pm 0.17$ & $4.10 \pm 0.17$ & $4.10 \pm 0.17$ \\
\hline & Arctic NE America & $4.24 \pm 0.12$ & $4.23 \pm 0.14$ & $4.22 \pm 0.15$ & $4.14 \pm 0.06$ & $4.13 \pm 0.05$ & $4.12 \pm 0.06$ & $4.14 \pm 0.15$ & $4.12 \pm 0.14$ & $4.12 \pm 0.13$ & $4.05 \pm 0.23$ & $4.03 \pm 0.20$ & $4.03 \pm 0.19$ \\
\hline
\end{tabular}

a See Table 3 for explanations of the scaling names.

Table 7

Global mean and statistical parameters for SLHL $P_{3}(n=23)$ and $P_{10}(n=31)$ as defined in CREp computed with 12 scaling procedures.

\begin{tabular}{|c|c|c|c|c|c|c|c|c|c|c|c|c|c|}
\hline Nuclide & Statistic & LAL-STD Musch & LAL-STD Lift & LAL-STD L & LAL-ERA Musch & LAL-ERALift & LAL-ERAL & LSD-STD Musch & LSD-STD Lift & LSD-STDL & LSD-ERA Musch & LSD-ERALift & LSD-ERAL \\
\hline \multirow[t]{7}{*}{$3 \mathrm{He}$} & Arithmetic mean & 121 & 118 & 116 & 122 & 119 & 117 & 123 & 120 & 117 & 125 & 121 & 119 \\
\hline & Weighted mean (Wm) & 121 & 118 & 116 & 122 & 120 & 118 & 122 & 119 & 117 & 124 & 121 & 119 \\
\hline & Error of the Wm & 3.17 & 3.41 & 3.57 & 2.62 & 2.87 & 3.03 & 2.68 & 2.84 & 3.00 & 2.32 & 2.42 & 2.50 \\
\hline & Weighted STDEV & 15 & 16 & 17 & 13 & 14 & 14 & 13 & 14 & 14 & 11 & 12 & 12 \\
\hline & Relative Wed STDEV ${ }^{\mathrm{b}}$ & $12.6 \%$ & $13.8 \%$ & $14.8 \%$ & $10.3 \%$ & $11.4 \%$ & $12.3 \%$ & $10.5 \%$ & $11.4 \%$ & $12.3 \%$ & $9.0 \%$ & $9.6 \%$ & $10.1 \%$ \\
\hline & Standard Deviation & 17 & 18 & 19 & 15 & 16 & 16 & 18 & 18 & 18 & 17 & 16 & 16 \\
\hline & MSWD $^{c}$ & 10.2 & 12.2 & 13.9 & 6.8 & 8.4 & 9.7 & 7.1 & 8.3 & 9.6 & 5.2 & 5.9 & 6.5 \\
\hline \multirow[t]{7}{*}{ 10Be } & Arithmetic mean & 4.16 & 4.13 & 4.11 & 4.18 & 4.15 & 4.13 & 4.10 & 4.05 & 4.00 & 4.14 & 4.09 & 4.03 \\
\hline & Weighted mean (Wm) & 4.15 & 4.13 & 4.09 & 4.13 & 4.10 & 4.07 & 4.14 & 4.10 & 4.01 & 4.11 & 4.08 & 3.99 \\
\hline & Error of the Wm & 0.04 & 0.04 & 0.04 & 0.04 & 0.04 & 0.04 & 0.04 & 0.04 & 0.04 & 0.05 & 0.04 & 0.04 \\
\hline & Weighted STDEV & 0.20 & 0.22 & 0.23 & 0.20 & 0.20 & 0.20 & 0.20 & 0.19 & 0.22 & 0.26 & 0.23 & 0.23 \\
\hline & Relative Wed STDEV & $4.9 \%$ & $5.2 \%$ & $5.7 \%$ & $4.8 \%$ & $4.8 \%$ & $4.9 \%$ & $4.8 \%$ & $4.7 \%$ & $5.5 \%$ & $6.3 \%$ & $5.6 \%$ & $5.6 \%$ \\
\hline & Standard Deviation & 0.27 & 0.29 & 0.30 & 0.26 & 0.26 & 0.26 & 0.23 & 0.24 & 0.27 & 0.28 & 0.25 & 0.24 \\
\hline & MSWD & 3.90 & 4.45 & 5.27 & 4.00 & 3.90 & 4.25 & 3.81 & 3.83 & 4.83 & 6.39 & 5.19 & 5.33 \\
\hline
\end{tabular}

Weighted Standard Deviation (see Appendix 1).

Weighted Standard Deviation/Weighted mean (see Appendix 1).

Mean Weighted Standard Deviation (see Appendix 1). 

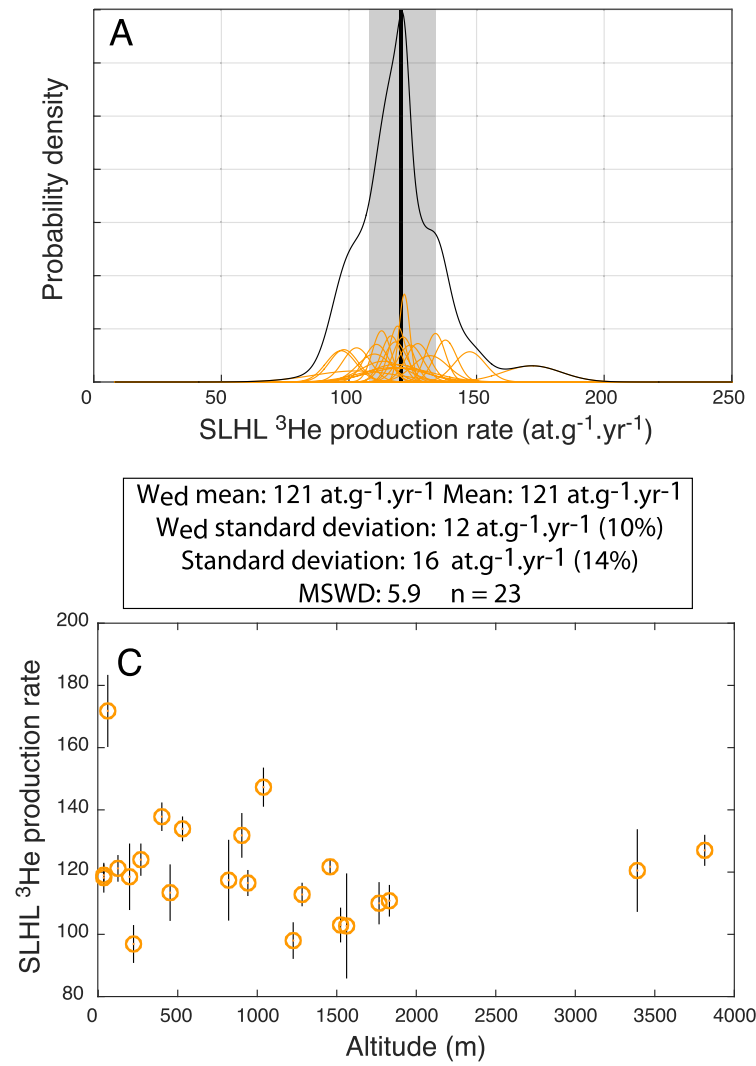

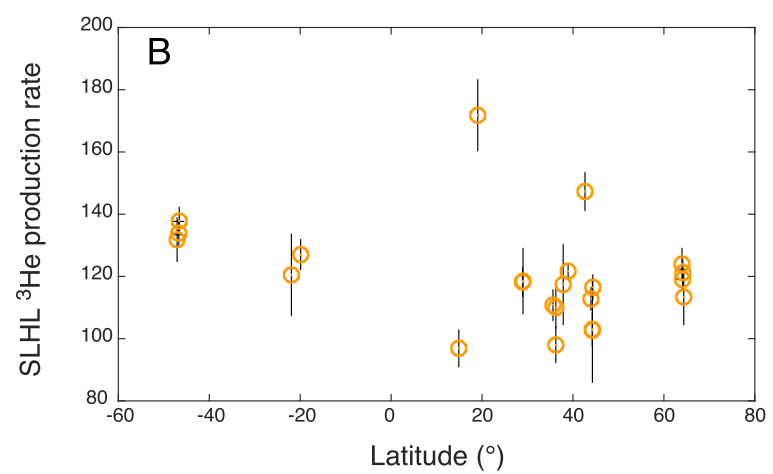

Scaling: LSD (Lifton et al., 2014)

Atmosphere: ERA 40 (Uppala et al., 2005) VDM: Lifton, 2016

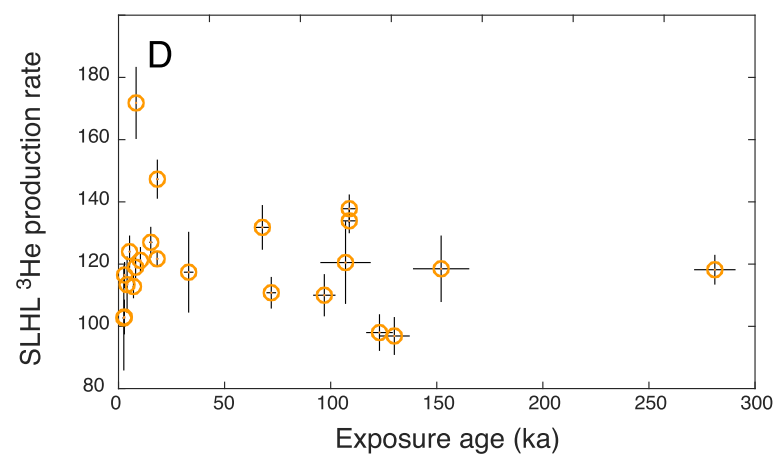

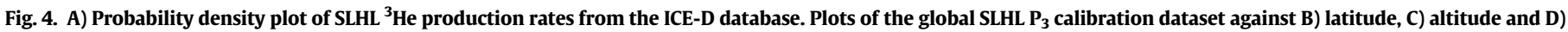
exposure age. These SLHL PR data are computed using the LSD-ERA-Lift scaling procedure.

account the spatial distribution of the present-day calibration dataset. Since several lithologies are not suited for both ${ }^{10} \mathrm{Be}$ and ${ }^{3} \mathrm{He}$, some regions are nuclides specific (e.g. basaltic terrains of Iceland and Hawaii are only defined for ${ }^{3} \mathrm{He}$ ). The boundaries of these regions will probably evolve in the future, with the addition of new calibration data.

\subsection{Calculation of global mean production rates}

The present version of CREp computes a global weighted average and the associated standard deviation taking into account all the existing site-specific production rates, without excluding any statistical outlier. This choice is motivated by the fact that a global average is supposed to represent the global scatter of the dataset, which may result from any source of variability (analytical, geological, inability of scaling scheme to adequately correct for spatial and temporal scaling). Also, rejection criteria are generally based on the assumption that the data are independent and normally distributed. Given the remaining different sources of error (scaling, geology), it is plausible that the actual distribution is not normal and that some inaccuracies are scaling dependent. Moreover, given the number of calibration sites in the existing dataset ( 31 calibration sites for ${ }^{10} \mathrm{Be}$ and 23 sites for ${ }^{3} \mathrm{He}$ ), the exclusion of outliers should not have a big effect.

\section{Discussion - production rates and uncertainties}

\subsection{Comparison with previous estimates}

The SLHL production rates compiled in CREp from the global
ICE-D database have values that are comparable with recent compilation (Borchers et al., 2016; Delunel et al., 2016; Heyman, 2014; Marrero et al., 2016). A detailed comparison of the resulting values is useless, however, since there are some differences in the data treatment and in the scaling framework used. Since the CREpICE-D tool proposes the largest existing calibration dataset, this study is a good opportunity to assess the efficiency of the different scaling frameworks by looking at the statistical dispersion of the computed SLHL rates.

\subsection{Dispersion of the world calibration database}

The efficiencies of 12 different scaling procedures (2 scaling schemes, 2 different atmospheric models and 3 geomagnetic databases) have been evaluated by calculating several statistical parameters. They were computed by taking into account all the individual SLHL production rates derived from the ICE-D global database, which represent 31 calibration sites for ${ }^{10} \mathrm{Be}$ and 23 sites for ${ }^{3} \mathrm{He}$.

Table 3 summarizes the 12 different scaling procedures that are tested here to scale the global $\mathrm{P}_{3}$ and $\mathrm{P}_{10}$ (respectively the ${ }^{3} \mathrm{He}$ and ${ }^{10} \mathrm{Be}$ production rates) calibration datasets of ICE-D.

Tables 4 and 5 displays all the $P_{3}(n=23)$ and $P_{10}(n=31)$ computed from the 12 different scaling procedures. Table 6 presents the regional $\mathrm{P}_{3}$ and $\mathrm{P}_{10}$ values as well as the calibration sites included in each region.

Table 7 shows the worldwide average $\mathrm{P}_{3}$ and $\mathrm{P}_{10}$ computed from the calibration data of ICE-D as well as the associated statistical parameters.

For the global dataset, computed statistical parameters are the 

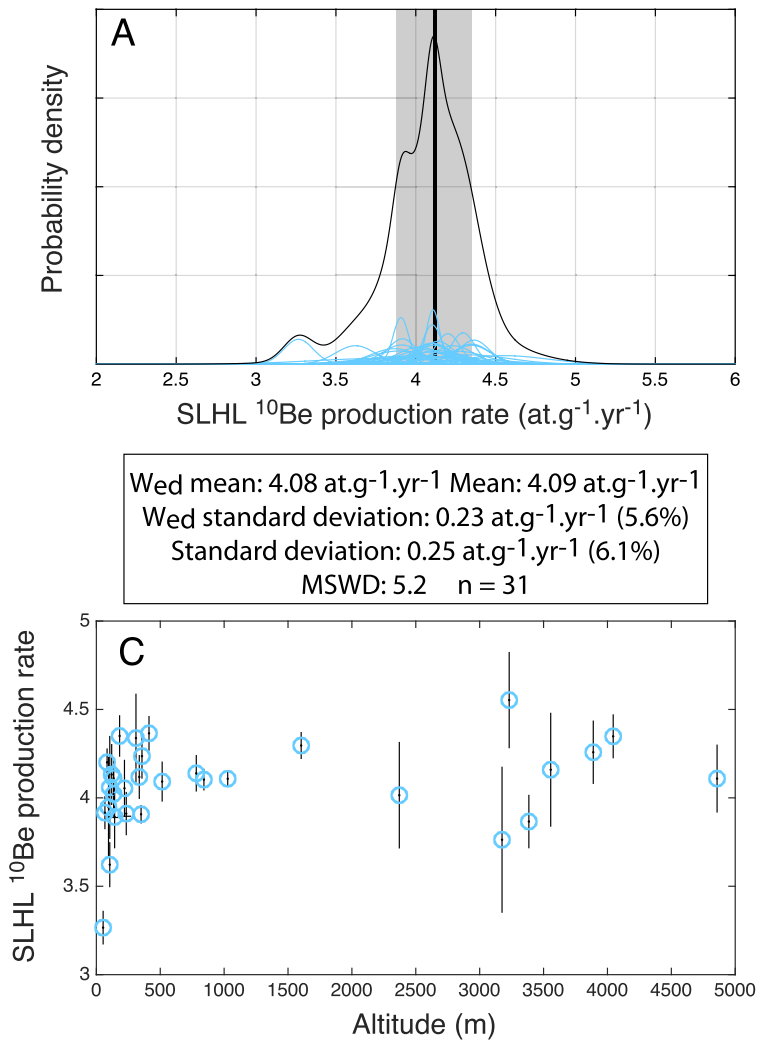

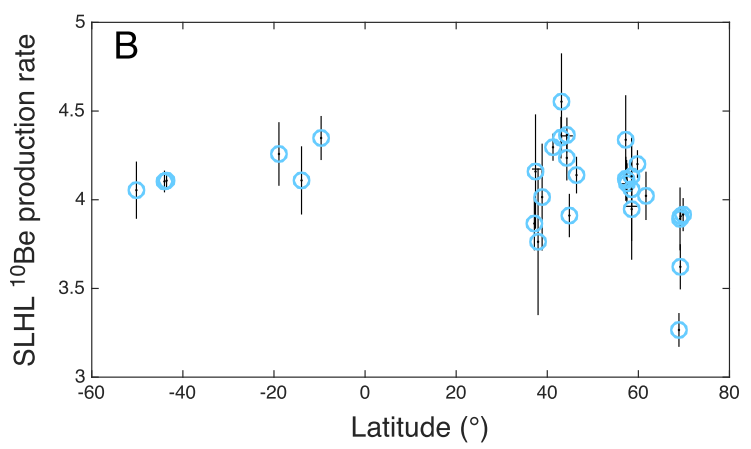

Scaling: LSD (Lifton et al., 2014)

Atmosphere: ERA 40 (Uppala et al., 2005)

VDM: Lifton, 2016

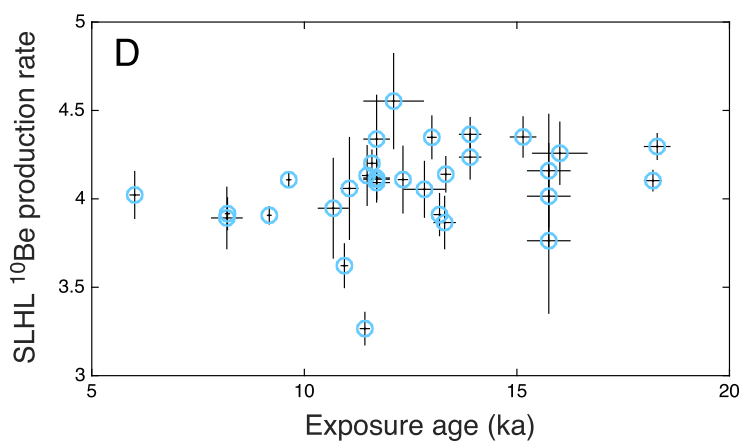

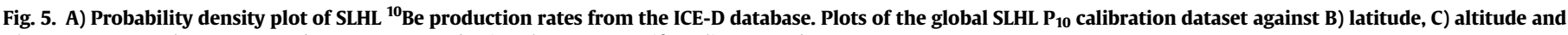
D) exposure age. These SLHL PR data are computed using the LSD-ERA-Lift scaling procedure.

MSWD, the weighted standard deviation and the standard deviation. MSWD is a good indicator of the over-dispersion relative to the uncertainties attached to each calibration site (Douglass et al., 2006; York, 1966). We thus use this as a first order indicator of the scaling ability to take into account the spatial and temporal variability of the cosmic-ray flux: the lower MSWD, the better the scaling procedure is.

In the case of the ${ }^{3} \mathrm{He}$ calibration database, MSWDs range from 5.2 to 13.9 , with corresponding relative weighted standard deviation of $9 \%$ and $15 \%$, respectively (Table 7 ). It is worth noting that the LSD model has lower MSWDs than the Lal/Stone model (mean value of 7 vs 10 , respectively) and thus seems to perform a better scaling. Similarly, the ERA40 atmosphere yields lower dispersion than the STD atmosphere.

In the case of ${ }^{10} \mathrm{Be}, \mathrm{MSWD}$ are lower than for ${ }^{3} \mathrm{He}$ : they range from 3.8 to 6.4 , for respective relative weighted standard deviation of $4.7 \%$ and $6.3 \%$. Contrary to what is observed with the ${ }^{3} \mathrm{He}$ database, the mean MSWDs of LSD and Lal/Stone are not significantly different (4.9 and 4.3, respectively) (Table 7). Surprisingly, the ERA40 atmosphere provides a better scaling than the STD atmosphere only when it is combined with the Lal/Stone model. Given the size of the dataset, this statement should however be considered with caution. For both ${ }^{3} \mathrm{He}$ and ${ }^{10} \mathrm{Be}$, the impact of the geomagnetic time-correction is quite limited and imperceptible (Figs. 4 and 5, Table 7), although the Atmospheric ${ }^{10} \mathrm{Be}$ VDM (Muscheler et al., 2005) and the Lifton VDM 2016 (Lifton, 2016) seems to provide slightly lower dispersions than the LSD geomagnetic framework (Lifton et al., 2014).

In order to evaluate possible weakness of these scaling procedures, we also tested potential correlation between the SLHL production rates of the database and the spatial position of the sites (latitude, longitude, altitude) (Figs. 4 and 5). These plots do not indicate any significant correlation between these spatial parameters and the calibrated production rates $\left(R^{2}\right.$ are lower than 0.14 in any case), supporting the idea that these 12 scaling procedures are not affected by any first order flaw; they can be considered as able to describe the main characteristics of the spatial variability of the cosmic ray flux on Earth, and notably the local peculiarities of the atmospheric pressure field or the non-dipole components of the geomagnetic field.

However, since large regions of the world do not contain any calibration site this statement may potentially be revised in the future, when new sites will be added in Asia and Africa (Fig. 2). Additionally, there is no significant trend between the scaled $\mathrm{P}_{3}$ and $\mathrm{P}_{10}$ and the age of the calibration surfaces.

\section{3. - Uncertainties and justification for choosing a global, a regional or a local production rate}

When a global production rate is selected, the dispersions of the global calibration datasets (evaluated by the weighted standard deviation) will yield exposure ages with minimum external $1 \sigma$ uncertainty of $5 \%$ for ${ }^{10} \mathrm{Be}$ and $10 \%$ in the case of ${ }^{3} \mathrm{He}$. Although this represents good progress compared to the previous global compilation (Balco et al., 2008 obtained $9 \%$ for ${ }^{10} \mathrm{Be}$ ), these uncertainties may remain quite high for certain applications, notably to perform high precision glacial chronologies. However, if the dated samples are relatively close to a well-calibrated region, it seems reasonable to prefer using a local or a regional production rate. This strategy has often been used since it minimizes potential scaling 
inaccuracies (e.g. Blard et al., 2014; Kaplan et al., 2011; Putnam et al., 2013), although it leaves open the potential for unrecognized regional or site-specific systematic geologic uncertainties, and the valid extent of the region in time and space is typically poorly defined. In this regard, it would also be preferable to select a calibration dataset whose exposure age is comparable to the age of the surface to be dated. In order to help users in choosing calibration sites having the most appropriate exposure ages, the selection map of CREp includes a function that allows filtering the age range of the calibration dataset.

On the other hand, if the sample to be dated is located more than $1000 \mathrm{~km}$ away from any calibration site, a warning sentence is displayed on the CREp interface to suggest choosing a global average. This is the case for Asia and Africa, continents where there is up to now no calibration data. CREp users are free to use their best judgement and test and use the scaling and production rate they consider most appropriate.

\section{Conclusion}

The CREp program is an online Octave/Matlab ${ }^{\odot}$ program (crep.crpg.cnrs-nancy.fr) that allows rapid and easy exposure age calculation for ${ }^{10} \mathrm{Be}$ and ${ }^{3} \mathrm{He}$ over the late Pleistocene. It offers a flexible parameterization of the scaling scheme and is linked to an online database, ICE-D, that includes the most recent calibration studies and developments in cosmic ray exposure ages. The Octave/ Matlab ${ }^{\odot}$ source code of CREp is available, making it easily amenable to further developments.

Further improvements could include the addition of ${ }^{21} \mathrm{Ne}$ and ${ }^{26} \mathrm{Al}$. New geomagnetic and atmospheric databases with improved accuracy will probably be added in the future. In this regard, a great improvement to CREp would be to add a time variable atmospheric pressure field, that could be, for example, derived from global circulation models (e.g. Staiger et al., 2007).

Since we designed the online calculator CREp and the ICE-D database to be constantly upgraded with the latest updates of cosmogenic nuclide systematics, we will greatly appreciate future readers' suggestions. Readers are notably invited to contact P.-H. Blard (blard@crpg.cnrs-nancy.fr) and G. Balco (balcs@berkeley. edu) to report any new production rate calibration study omitted in ICE-D.

\section{Acknowledgments}

This work was funded by the INSU EVE-LEFE program and the ANR Jeunes Chercheurs GALAC project "ANR-11-JS56-011-01". G. Balco's contribution to this work was supported in part by the Ann and Gordon Getty Foundation. J. Charreau, R. Pik, D. Bourlès, J.-F. Ritz, M. Ferry, V. Jomelli, D. Brunstein provided very useful advice about the design of CREp. R. Braucher and J. Amalberti are thanked for useful discussions about muonic production scaling and Matlab $\mathrm{GUI}^{\odot}$, respectively. This is CRPG contribution $n^{\circ} 2468$.

\section{Appendix A. Supplementary data}

Supplementary data related to this article can be found at http:// dx.doi.org/10.1016/j.quageo.2016.11.006.

\section{Appendix 1. Definition of the statistical parameters}

Given a set of $\mathrm{N} \mathrm{x}_{\mathrm{i}}$ measurements with their respective uncertainties $\sigma_{\mathrm{i}}$ :
- MSWD: Mean Squared Weighted Deviation (or reduced $\mathrm{Chi}^{2}$ )

$M S W D=\frac{1}{N-1} \sum_{i=1}^{N} w_{i}\left(x_{i}-x_{\text {mean }}\right)^{2}$

with $\mathrm{w}_{\mathrm{i}}=1 / \sigma_{\mathrm{i}}^{2}, \mathrm{~N}$ the number of variable and $\mathrm{x}_{\text {mean }}$ the arithmetic mean of the dataset.

* if MSWD $>1$, the dataset is over-dispersed regarding the individual analytical uncertainties, suggesting the existence of unrecognized external sources of uncertainty.

* if MSWD $<1$, the dataset is over-dispersed regarding the analytical uncertainty, suggesting that uncertainties are overestimated.

- Weighted-mean $x_{m}$

$x_{m}=\frac{\sum_{i=1}^{N} w_{i} x_{i}}{\sum_{i=1}^{N} w_{i}}$

- Error of the weighted-mean $\sigma_{\mathrm{w}}$

$\sigma_{w}=\sqrt{\frac{1}{\sum_{i=1}^{N} w_{i}}}$

- Weighted standard deviation Wed_STD

Wed_STD $=\sqrt{\frac{N \sum_{i=1}^{N} w_{i}\left(x_{i}-x_{m}\right)^{2}}{(N-1) \sum_{i=1}^{N} w_{i}}}$

\section{Appendix 2. Tutorial of the CREp online calculator}

\section{Step1: Initial parameterization}

The parameterization commands are presented in Fig. A1.

The initial parameterization is done thanks to radio buttons, making this choice quick and easy. Four items have to be set by the users (Fig. A1, see Section 2 for detailed presentation):

* Nuclide: ${ }^{10} \mathrm{Be}$ or ${ }^{3} \mathrm{He}$

* Scaling scheme: Lal-Stone time corrected (Balco et al., 2008; Lal, 1991; Stone, 2000) or LSD (Lifton et al., 2014).

* Atmosphere model: ERA-40 (Uppala et al., 2005) or the U.S. standard atmosphere (National Oceanic and Atmospheric Administration, 1976).

* Geomagnetic database: Muscheler et al. (2005), Lifton (2016) or the LSD geomagnetic framework (Lifton et al., 2014).

Users may also input their own Virtual Dipole Moment database in the programme by clicking to "Browse your own data". The input Virtual Dipole moment data should be presented in two columns. The right column should contain the ages in kyr and the second one the Virtual Dipole Moment values in $10^{22} \mathrm{~A} \mathrm{~m}^{2}$. Hence, the present value of $7.746 \times 10^{22} \mathrm{~A} \mathrm{~m}^{2}$ (Finlay et al., 2010) should be input 7.746.

When the geomagnetic reconstruction is selected or correctly imported, it is plotted on the interface. Once the parameters have been selected, users need to push the "Next" button. The scaling parameterization is then locked. 
2. Step2: Upload of the samples characteristics of SLHL production rate

This step consists in importing the cosmogenic nuclides data and the characteristics of the samples to be dated (Fig. A2). The input data file must be in the format of an Excel (c) spreadsheet (xls or xlsx). A template can be downloaded using the "Download template" button.

The uploaded table must include the following data (similar information is also available by clicking the "Instructions for Sample File" button):

Column 1: Sample name.

Column 2: Latitude (Decimal degrees). Range from -90 to $90^{\circ}$. Negative value for Southern Hemisphere.

Column 3: Longitude (Decimal degrees). Range from -180 to $180^{\circ}$. Negative value for Western Hemisphere.

Column 4: Altitude (masl).

Column 5: Cosmogenic nuclide concentration (at $\mathrm{g}^{-1}$ ). IMPORTANT: For ${ }^{10} \mathrm{Be}$, use the 07KNSTD standardization (Nishiizumi et al., 2007). If the ${ }^{10} \mathrm{Be}$ concentrations are computed using another standardization, convert them to 07KNSTD before loading your data in CREp (see http://hess.ess.washington.edu/math/docs/al_ be_v22/al_be_docs.html for more details).

Column 6: Analytical 1-sigma uncertainty (at $\mathrm{g}^{-1}$ ).

Column 7: Shielding correction (dimensionless). Range from 0 to

1.

Column 8: Sample density $\left(\mathrm{g} \mathrm{cm}^{-3}\right)$.

Column 9: Sample thickness $(\mathrm{cm})$.

Column 10: Erosion $\left(\mathrm{cm} \mathrm{yr}^{-1}\right)$.

Once the sample characteristics are loaded, users have to press the "Next" button.

\section{Step3: Selection of SLHL production rate}

Users may choose i) a world wide mean, ii) a local or a regional rate, or iii) enter their own calibration data (Fig. A3). For this choice, users just have to check the relevant button.

If the option "Local and Regional Values" is checked, a selection map is displayed, showing all the calibration sites currently available in ICE-D (Fig. A4).

Users may choose one or more single calibration sites by clicking on the corresponding tag. The selected sites appear in red. If more than one site is selected, CREp computes a weighted mean and its associated uncertainty.

Alternatively, the "Region View" button may be activated. In this case, users may choose one of the pre-calculated regional average production rates.

If users choose to import their own SLHL production rate, they can either load directly a SLHL production rate value (Note, however, that, in that case, user must ensure that this value is computed with the appropriate scaling scheme and parameters), or input data from a calibration surface to compute a new SLHL production rate. The input fields are (Fig. A5):

- Nuclide concentrations with the one-sigma uncertainty in at $\mathrm{g}^{-1}$. For dataset with multiple exposure samples, outlier selection, thickness correction, altitude scaling and calculation of a global average need to be computed before entering this average concentration value.

- Independent age with its one-sigma uncertainty in kyr.

- Geographic location: latitude and longitude in decimal degrees (negative values for Southern and Western location).

- Altitude in masl.

- Erosion rate in $\mathrm{cm} \mathrm{yr}^{-1}$.
Once these data are entered, users have to click on the "Calculate PR" button and the computed SLHL then appears in a window called "Display your Production Rate (at/g/yr)".

Whatever the options, the value of the currently selected production rate is always displayed in a window located at the bottom of the page (Fig. A6).

Finally, once the user is happy with the PR selection, she/he has to click the "Calculate" button.

\section{Step 4: Results display and export}

The computed ages appear by default in the form of a Table that can be exported as a.xlsx file by clicking on the relevant button (Fig. A7). Note that two types of uncertainties are provided along with the ages: with and without the error arising from the production rate. In the second case ( $1 \sigma$ without PR error), only analytical errors are included. These uncertainties should be used to test the statistical dispersion of objects belonging to the same surface. It is also possible to display the computed ages in the form of density probablity plots (Fig. A8).

\section{Note on input data}

Input data for the CREp program are Excel ${ }^{\circledR}$ spreadsheet in.xls or.xlsx format. Make sure there is only one page in the file or that your data are on the first page. Numeric values should form a rectangular block without empty cells in it. Be aware that the number format impact the value read by the CREp program: the program considers the number of significant figures that is displayed, and not the double precision value. This may lead to a loss of precision. For example, if the scientific format with only one digit is used to display a nuclide concentration of 525600 at $^{-1}$, the CREp value will round this value to 530000 at $^{-1}$. The documents have to be placed inside the CREp folder. Two types of data may be input: the characteristics of the samples to be dated and the Virtual Axial Dipole Moment values. Only the prior is mandatory.

\section{Proper credit to previous publications}

It is important to give proper credit to the researchers that produced the original calibration data as well as the scaling schemes. Thus, CREp automatically generates a relevant publication list that should be quoted, taking into account the references corresponding to the used scaling procedure and the calibration dataset in use.

In the output Excel file (3rd spreadsheet named "References"), CREp automatically generates a text describing the scaling and the production rates used in the calculation. This text also includes an exhaustive list of references that need to be quoted. In its current version, CREp generates the following text (as well as the full list of the quoted references). It can be pasted as it is in any publication that used CREp, for example:

"These cosmogenic ${ }^{10} \mathrm{Be}$ exposure ages were calculated on Fri Oct 14 2016, using the online CREp calculator (crep.crpg.cnrsnancy.fr; Martin et al., 2016). They were computed using the scaling scheme Lal/Stone time dependent (Balco et al., 2008; Lal, 1991; Stone, 2000), with the ERA-40 atmosphere model (Uppala et al., 2005), the geomagnetic record of Lifton 2016 VDM (Laj et al., 2004; Lifton, 2016; Pavón-Carrasco et al., 2014; Ziegler et al., 2011) and the production rates calibrated by Kaplan et al. (2011); Kelly et al. (2015); Martin et al. (2015)."

\section{Appendix 3. Tutorial of the CREp Matlab (c) program}

The stand alone Matlab version of CREp is designed in a similar 
fashion than the online calculator (crep.crpg.cnrs-nancy.fr), with these 2 exceptions: i) in the Matlab version of CREp, the samples characteristics are loaded after the choice of the production rate and ii) in the Matlab version, the PR selection is done from a list, the interactive map is not available.

\section{Installation of the CREp program on the user's computer}

The CREp program has been developed with the Matlab ${ }^{\odot} 2012 \mathrm{~b}$ version. To start using the CREp program, users have to follow these steps:

- Download the CREp folder. It contains the CREp program, along with the CREp graphical user interface file, a functions folder and a constants folder. Two trial .xls files are proposed to test geomagnetic data importation and exposure age calculation.

- Launch Matlab ${ }^{\circledR}$ and make the CREp folder your current directory.

- In the Matlab Current Folder window, right-click on the CREp7.m file and select Run. The CREp program window will open. If elements are overlapping, or if the window is too small, resize it.

\section{Step1: Initial parameterization}

The parameterization commands are presented in Fig. B1.

The initial parameterization is done thanks to radio buttons, making this choice quick and easy. Four items have to be set by the users (Fig. B1, see Section 2 for detailed presentation):

- Nuclide: ${ }^{10} \mathrm{Be}$ or ${ }^{3} \mathrm{He}$

- Scaling scheme: Lal-Stone time corrected (Balco et al., 2008; Lal, 1991; Stone, 2000) or LSD (Lifton et al., 2014).

- Atmosphere model: ERA-40 (Uppala et al., 2005) or the U.S. standard atmosphere (National Oceanic and Atmospheric Administration, 1976).

- Geomagnetic database: Muscheler et al. (2005), Lifton (2016) or the LSD geomagnetic framework (Lifton et al., 2014).

Users may also input their own Virtual Dipole Moment database in the programme by clicking to "Browse your own data". The imported data have to be placed in the CREp folder and require a specific format described in Section 3.3. When the geomagnetic reconstruction is selected or correctly imported, it is plotted on the interface.

Once the parameters have been selected, users need to push the "Next" button. The scaling parameterization is then locked.

\section{Step2: selection of SLHL production rate}

This interface is presented on Fig. B2.

After the selection of the parameters, the CREp program computes a reference SLHL production rate. This reference production rate may come from the calibration dataset provided with the CREp program, but users may as well use their own calibration data. This choice is done using the first radio button panel (Fig. B2).

If the users decide to use the production rates proposed in the program, they have several possibilities: either a worldwide average value can be chosen, a regional value, or a variable number of calibration datasets can be selected and used to calculate a weighted average. A specific interface box permits this selection (Fig. B2). In the online version of CREp, this selection is done through an interactive map. When the calibration dataset is selected, the user has to press the "Display PR" button and a SLHL production rate value then appears with the associated one-sigma uncertainty and scaling factor.

At that point, users can compute other production rates for the same scaling scheme in order to compare them. By doing so, the first production rate will not be lost, and the computed production rates will accumulate in the related window. When two or more production rates are computed, users can choose to compute a weighted mean out of them by pressing the "weighted mean" button. To erase these selected production rates selection, one may click on the "Clear" button.

Importantly, it is necessary to push the "Load for calculation" button to export the selected production rate and load this for calculating the ages. The selected value will appear in red in the "Current SLHL PR" box located in the bottom right corner. Then, the "Next" button become active and can be pushed to move to the next step.

If users choose to import their own SLHL production rate, they can either load a SLHL production rate value directly, or input several data from a calibration surface to compute a new SLHL production rate. The input fields are:

- Nuclide concentrations with the one-sigma uncertainty in at. $^{-1}$. For datasets with multiple exposure samples, outlier selection, thickness correction, altitude scaling and calculation of a global average need to be computed before entering this average concentration value.

- Independent age with its one-sigma uncertainty in kyr.

- Geographic location: latitude and longitude in decimal degrees (negative values for Southern and Western location).

- Altitude in masl.

- Erosion rate in $\mathrm{cm} \mathrm{yr}^{-1}$.

The last filled field has to be validated using Tab or Enter to enable the "Display PR" button. If the inputs do not have the required format, they will not be accepted. When all fields are correctly filled, the "Display PR" has to be pushed to enable the production rate calculation.

An alternative possibility is to enter a SLHL production rate along with its uncertainty directly. Note, however, that, in that case, user must ensure that this value is computed with the appropriate scaling scheme and parameters.

Once again, it is necessary to finally push the "Load for calculation" button to export the selected production rate and load this for calculating the ages. The selected value will appear in red in the "Current SLHL PR" box located in the bottom right corner. The "Next" button becomes active and can then be pushed to move to the next step.

\section{Step 3: Data importation and exposure age calculation}

This last step consists in importing the cosmogenic nuclide data and the characteristics of the samples to be dated. The input data file must be in the format of an Excel $^{\odot}$ spreadsheet (xls or xlsx) and has to be placed in the CREp folder. The spreadsheet must include the following data (Users may use the available spreadsheet called "Trial10BeData.xlsx" as a template):

Column 1: Sample name.

Column 2: Latitude (Decimal degrees). Range from -90 to $90^{\circ}$. Negative value for Southern Hemisphere.

Column 3: Longitude (Decimal degrees). Range from -180 to $180^{\circ}$. Negative value for Western Hemisphere. 
Column 4: Altitude (masl).

Column 5: Cosmogenic nuclide concentration (at. ${ }^{-1}$ ). IMPORTANT: For ${ }^{10} \mathrm{Be}$, use the 07KNSTD standardization (Nishiizumi et al., 2007). If the ${ }^{10} \mathrm{Be}$ concentrations are computed using another standardization, convert them to 07KNSTD before loading your data in CREp (see http://hess.ess.washington.edu/math/docs/al_ be_v22/al_be_docs.html for more details).

Column 6: Analytical 1-sigma uncertainty (at.g ${ }^{-1}$ ).

Column 7: Shielding correction (dimensionless). Range from 0 to 1.

Column 8: Sample density $\left(\mathrm{g} . \mathrm{cm}^{-3}\right)$.

Column 9: Sample thickness $(\mathrm{cm})$.

Column 10: Erosion $\left(\mathrm{cm}_{\mathrm{yr}} \mathrm{r}^{-1}\right)$.

If the importation is successful, the name of the imported file appears in the "Imported Data" box and the "Calculate" button is enabled.

Clicking on the "Calculate" button launch the calculation procedure. This step may last several seconds or minutes, depending on the number of calculable ages provided in the input data file. The calculation for one age should not exceed $3 \mathrm{~s}$, except if the user imported a very high-resolution geomagnetic reconstruction (time step smaller than $50 \mathrm{yr}$ ). A wait bar informs the users of the calculation progress.

\section{Results display}

Results can be displayed in three different ways using the radio button panel "Results Display" that appears after the calculation. With the "PDF (Graphic)" mode (Fig. B3), users can display a graph with the different probability density functions (PDF) associated with each age. With the "Ages" mode, a table presents the ages and associated uncertainties (Fig. B4). The "PDF (Table)" mode provides a table that contains the numerical values of each density functions. By default, the CREp program shows the density function graph.

Results presented with the "PDF" (Probability Density Function) modes and those of the "Ages" mode are not presented with the same uncertainties. The uncertainties of the "PDF" results include the analytical uncertainties only, while the "Ages" table provides both the analytical uncertainties and the total uncertainties. Total uncertainties include both the analytical uncertainties and the production rate uncertainties. They correspond to more conservative ages of the samples. Thus these results are useful to investigate the dispersion of the different ages obtained for the same object. For example, the total uncertainties should be used to test the potential impact of erosion or inheritance on a pool of ages from the same moraine.

It is important to note that the ability of the CREp program to compute an exposure age depends on the time steps used in the program and of the length of the geomagnetic reconstruction relatively to the age of the sample. Therefore, a sample can be too young for the program or too old for the geomagnetic database. To visualise potential problems with too young or too old ages, users may select the "Age" result display mode. The displayed table contains a "Status" column. If the status is "OK", the age calculation performed successfully and a probability density function is available for the sample. If a different status is posted, it is impossible for the program to provide a density function and three situations may be covered:

- The status is "Sample too old for geomagnetic database". The database covers too narrow a time span. The program cannot calculate the geomagnetic time correction and no age will be proposed for the sample.

- The status is "Sample too young to provide probability density function". Given the time steps used by the program, the time correction is conducted without using probability density function.

- The status is "Sample too old or too young to provide probability density function". Given the time steps used by the program, the time correction is conducted without using probability density function. Another possibility is that the age of the sample is approximately the oldest age of the geomagnetic reconstruction, the time correction is conducted without using probability density function. The two classes of ages that do not include probability density functions are indicative ages and should not be used to discuss and establish the age of a geological object.

The result interface and the different possible status are presented in Fig. B3.

The numeric results displayed can be copied and pasted into a spreadsheet document but it is also possible to directly export them into spreadsheet format by clicking on the "Export" buttons above the "Results" box. Users can export the ages and/or the probability density functions of the ages directly.

\section{Results export}

The CREp program allows the exportation of the computed data thanks to the "Export" buttons. Depending on the Matlab version and the platform (Mac/PC) of the users, export may produce an Excel $^{\odot}$ document (.xls or.xlsx) or a standard.csv document. For better compatibility between different Matlab ${ }^{\odot}$ versions and platforms, the exported data are merely blocks of numerical values without text indications.

When the "Ages" table is exported, the data block produced contains 3 columns and one line per dated sample. The first line gives the scaling factor, the second one the Age and the third, the one-sigma uncertainty associated with the age. The ages are calculated in the order of appearance of the input spreadsheet so this block can be copied and pasted on the right of the input datablock and the ages will match the good samples. If no age was calculated for the sample (see Section 5), the corresponding fields will indicate "NaN".

When the PDF table is exported, the first column of the data block is a time vector on which all the calculated PDF are defined on a seven-sigmas interval. Each other column corresponds to the age PDF of a sample. Only samples with the "OK" status have a PDF vector in the data block. To identify each PDF, users just have to remove samples with an alternative status from the sample list. The name order in the resulting list will correspond to the PDF order of the table.

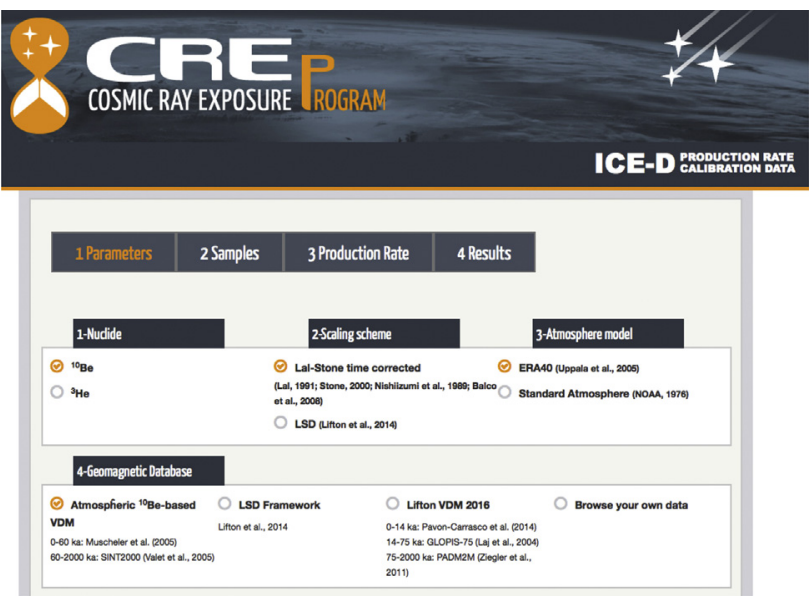

Fig. A1. Step 1: choice of the scaling parameters. 


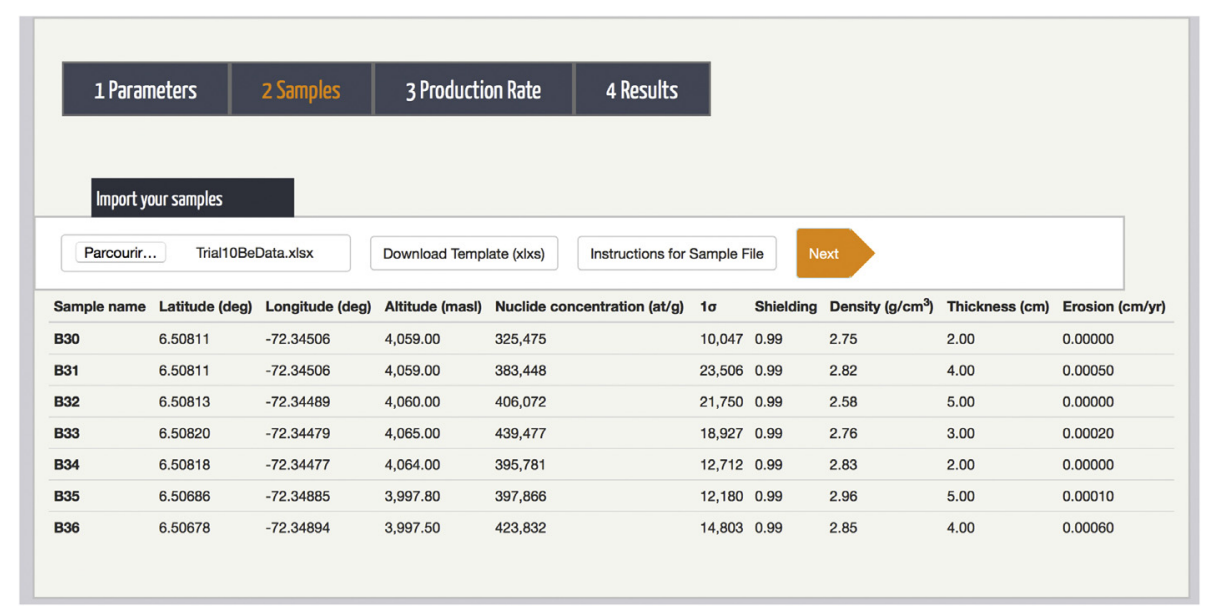

Fig. A2. Step 2. Importation of the samples characteristics.

\begin{tabular}{|c|c|c|c|c|}
\hline 1 Parameters & 2 Samples & 3 Production Rate & 4 Results & \\
\hline \multicolumn{5}{|l|}{ 1-Production Rate } \\
\hline World wide mean & & (- Local and Regi & Values & My Values \\
\hline
\end{tabular}

Fig. A3. Selection of the type of production rate.

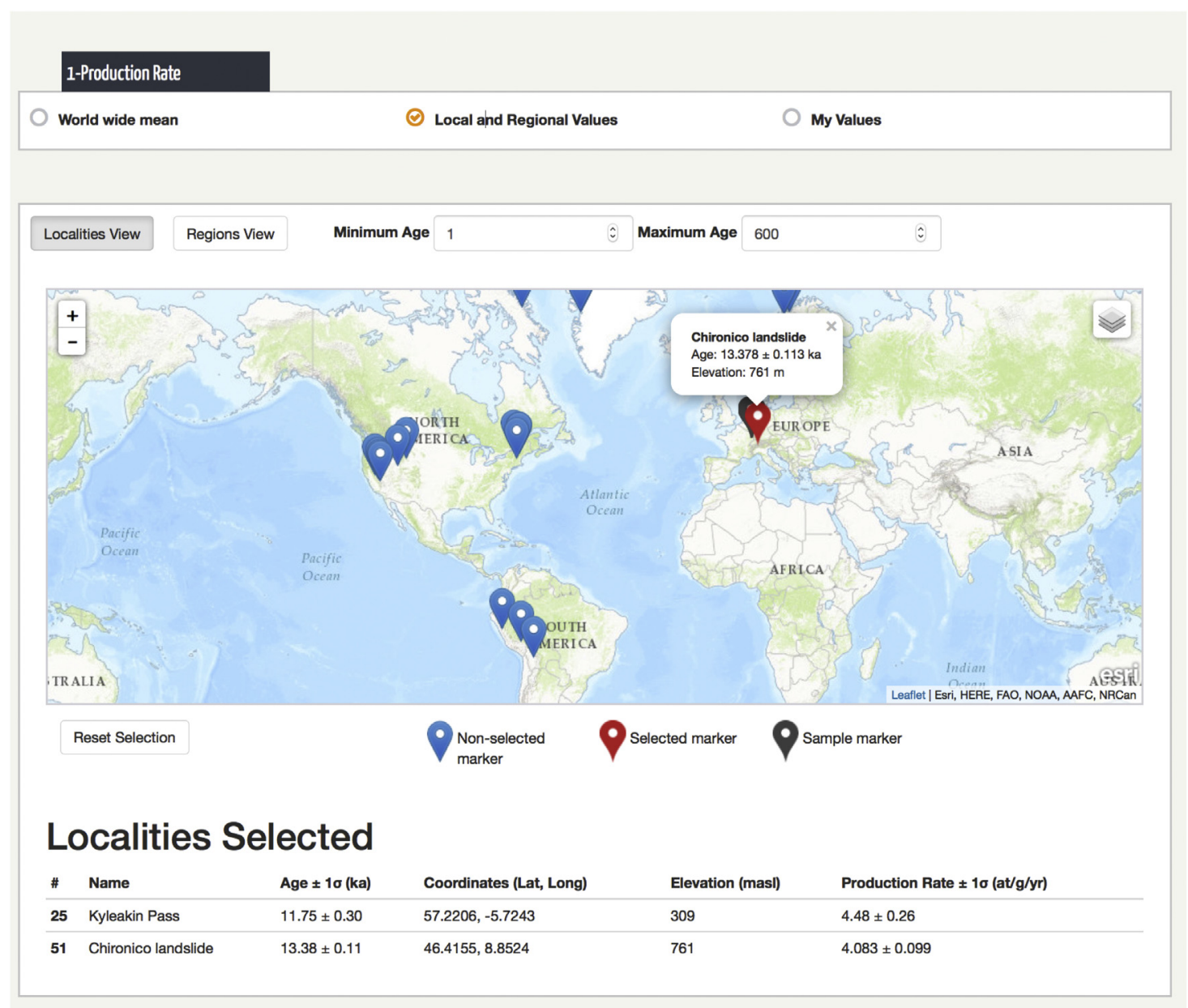

Fig. A4. CREp interface map for selecting local or regional calibration sites. 


\section{2-My Values}

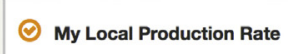

My SLHL Production Rate

\begin{tabular}{|l|}
\hline \multicolumn{1}{|c|}{ Age (ka) } \\
\hline 18.2 \\
\hline 288000 \\
\hline \multicolumn{2}{|c|}{ Conc (at/g) } \\
\hline \multicolumn{2}{|c|}{ Latitude } \\
\hline 2.15 \\
\hline 2846 \\
\hline
\end{tabular}

\begin{tabular}{|l|l|}
\hline \multicolumn{1}{|c|}{$\mathbf{1 \sigma ( k a )}$} \\
\hline 0.2 & \\
\hline \multicolumn{1}{|c|}{$\mathbf{1 \sigma ( a t / g )}$} \\
\hline 21000 \\
\hline \multicolumn{2}{|c|}{ Longitude } \\
\hline 2.16 \\
\hline Erosion Rate (cm/year) \\
\hline 0 \\
\hline
\end{tabular}

3-Display your Production Rate (at/g/yr)

\section{4-Calculate PR}

\begin{tabular}{lll}
\hline Scaling Fact. & SLHL & 10 \\
\hline 3.994970742 & 3.98 & 0.29 \\
\hline
\end{tabular}

Load for Calculation

Fig. A5. CREp interface for computing user's own production rates.

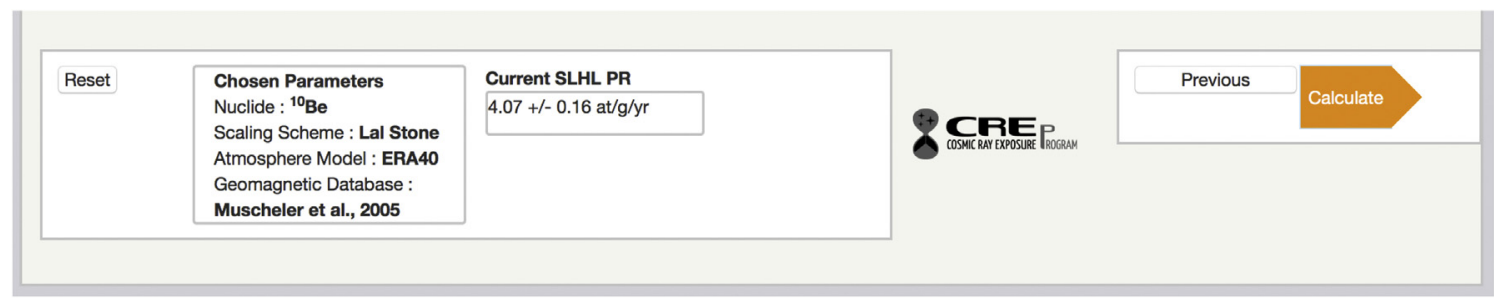

Fig. A6. Display of the currently selected SLHL production rate and corresponding scaling framework.

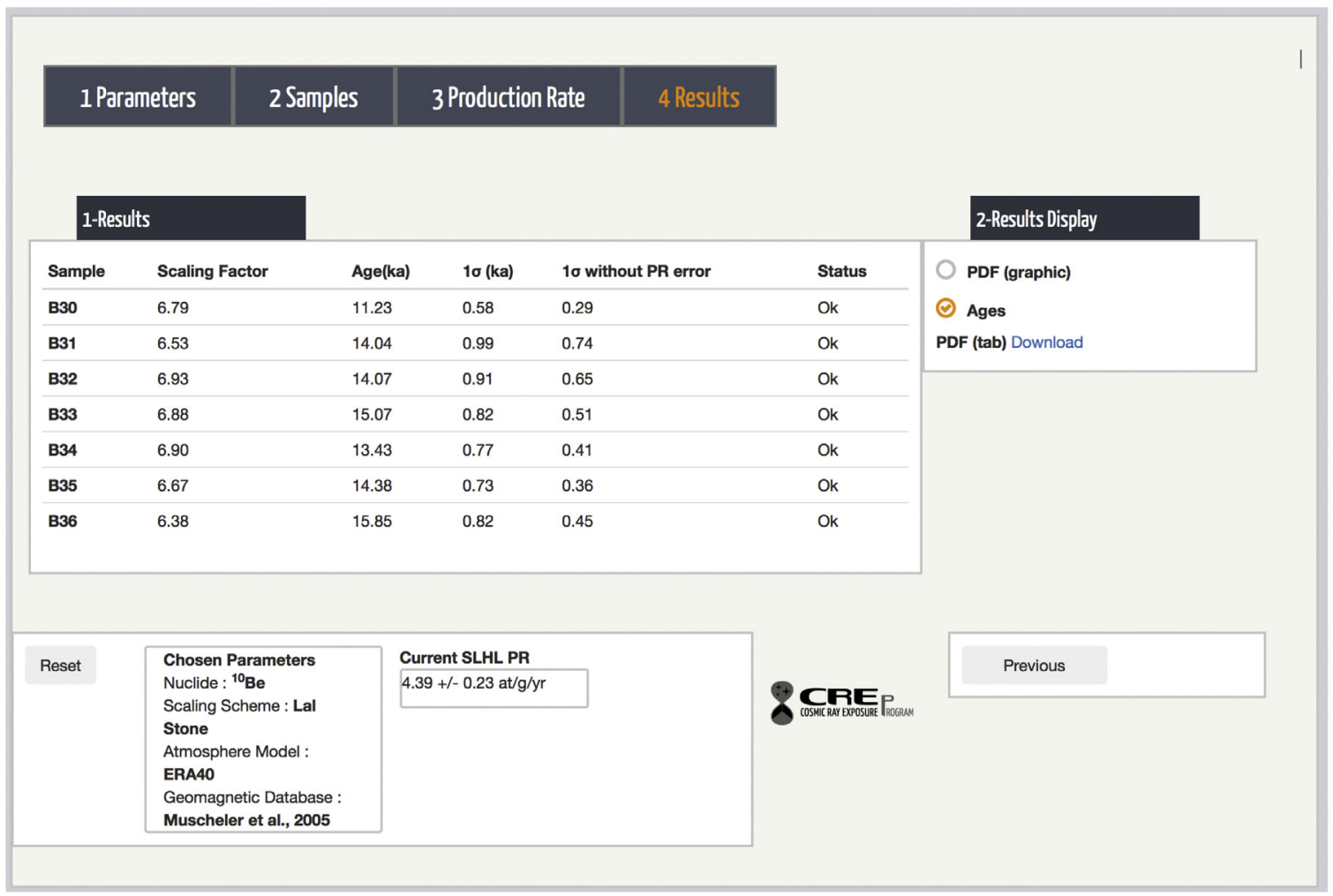

Fig. A7. Results display in the form of a table. 


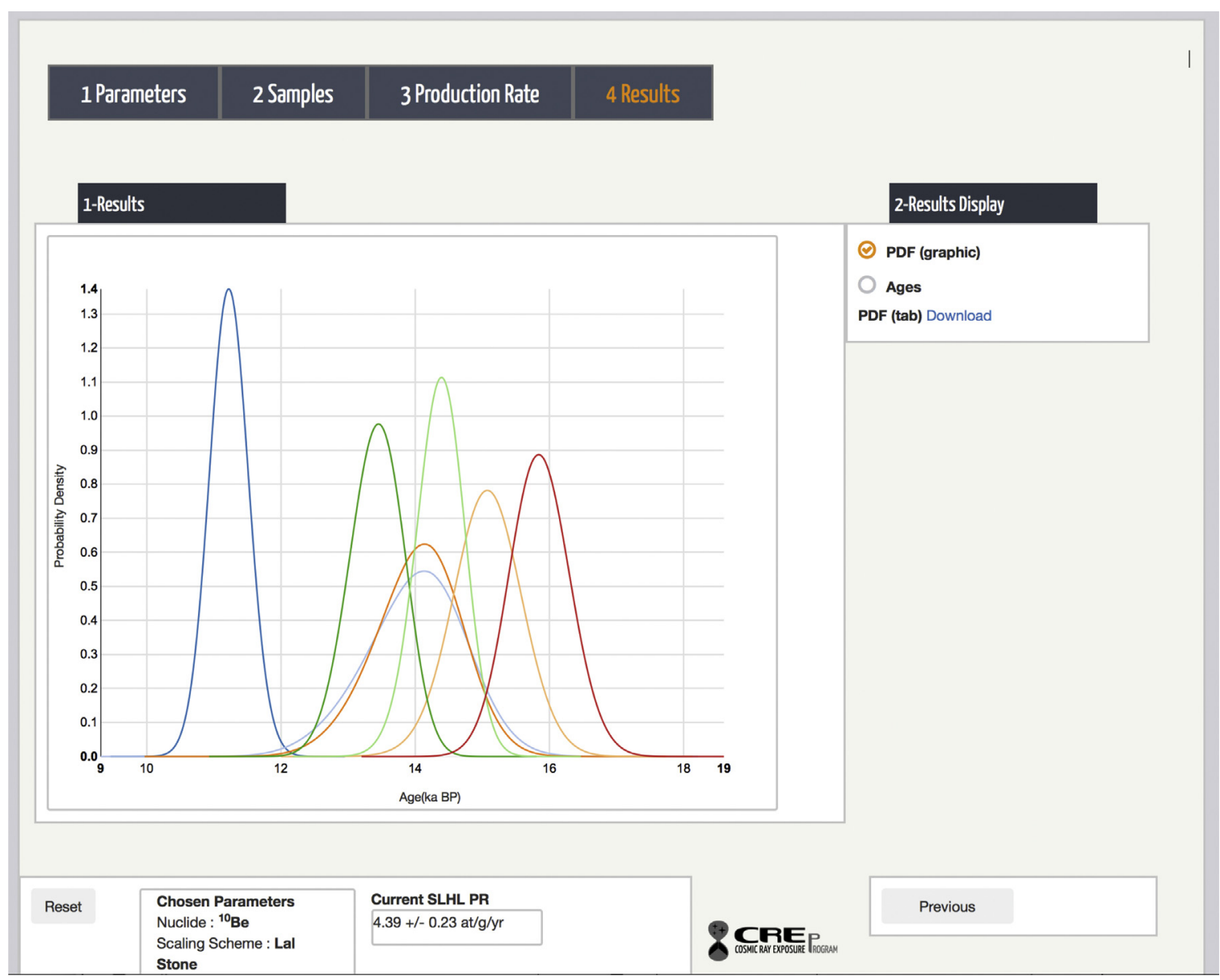

Fig. A8. Results display in the form of a probability density plot.

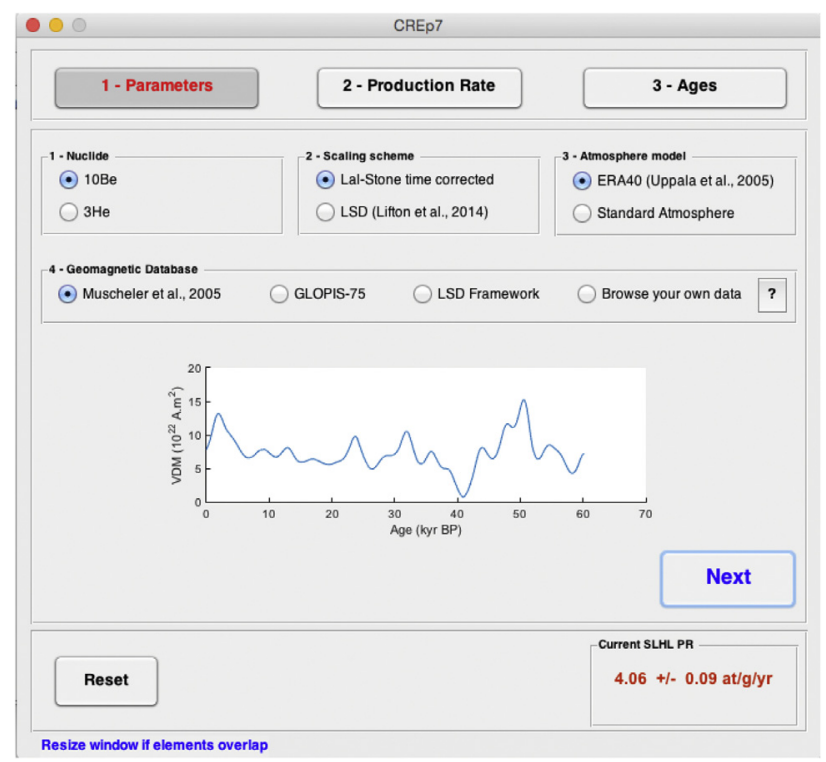

Fig. B1. Step 1: choice of the parameters. 


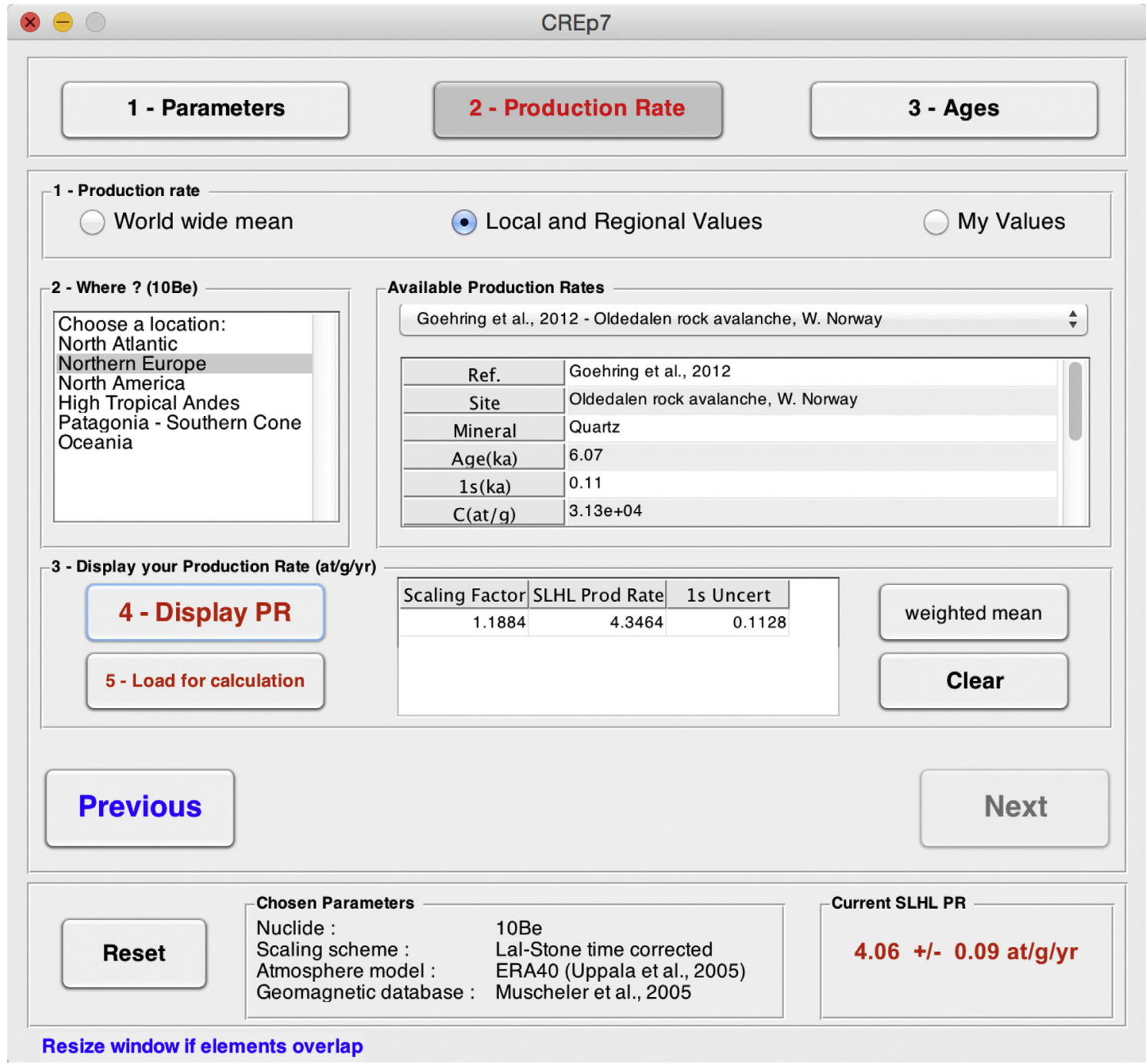

Fig. B2. Selection of a calibration dataset and calculation of SLHL production rate. 


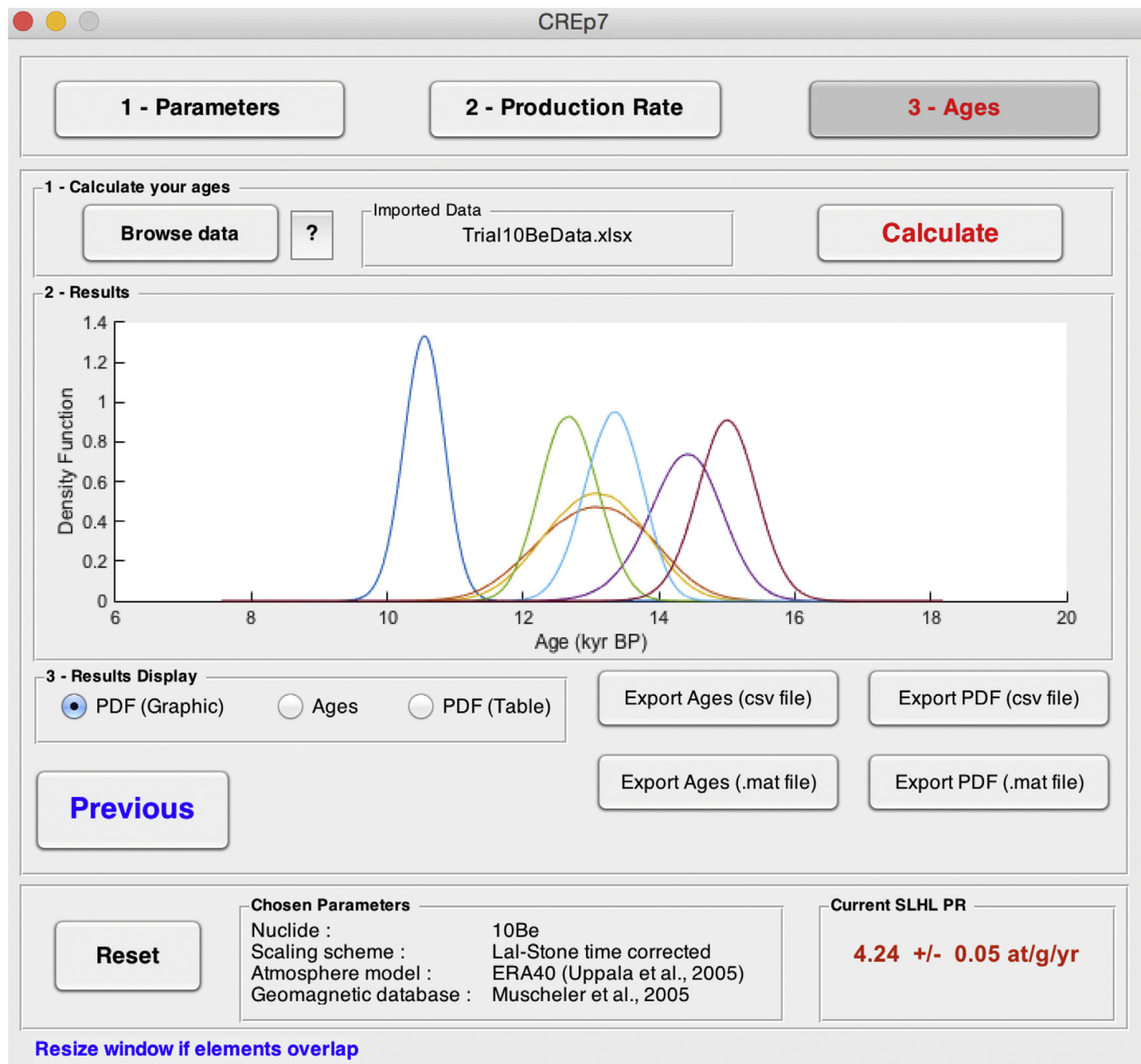

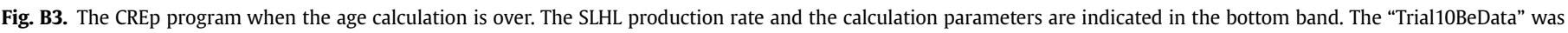
successfully imported, the "Calculate" button, was clicked. The "PDF (Graphic)" mode is selected for result display. The export buttons can be clicked to save the results.

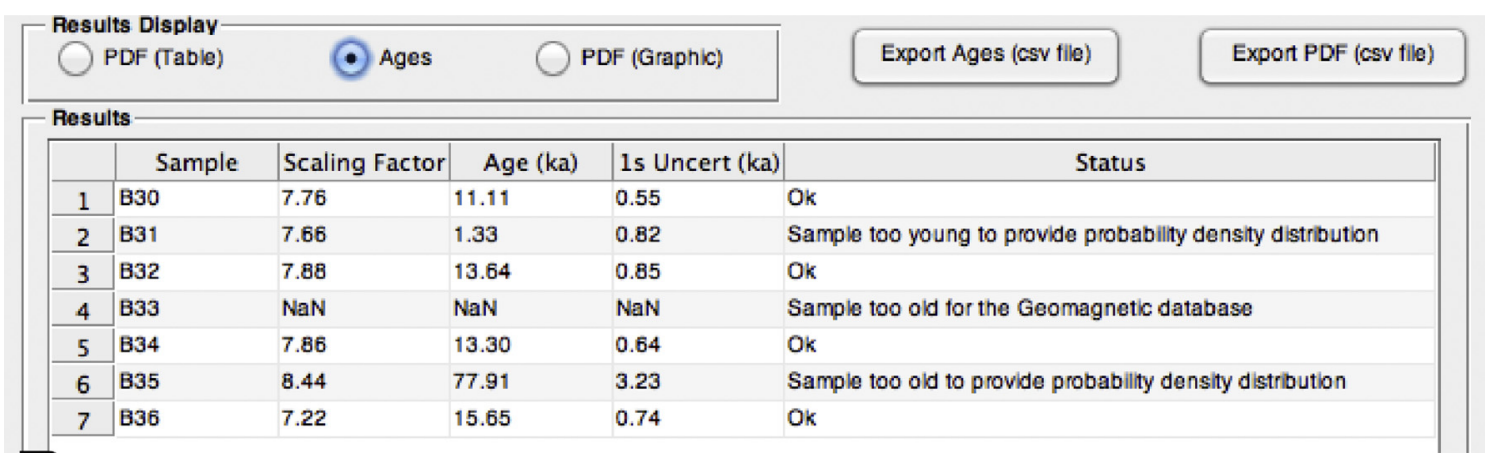

Fig. B4. Results display with various status for the calculated ages. 


\section{References}

Ackert, R.P., Singer, B.S., Guillou, H., Kaplan, M.R., Kurz, M.D., 2003. Long-term cosmogenic ${ }^{3} \mathrm{He}$ production rates from $40 \mathrm{Ar} / 39 \mathrm{Ar}$ and $\mathrm{K}-\mathrm{Ar}$ dated Patagonian lava flows at $47^{\circ} \mathrm{S}$. Earth Planet. Sci. Lett. 210, 119-136. http://dx.doi.org/ 10.1016/S0012-821X(03)00134-1.

Amidon, W.H., Farley, K.A., 2011. Cosmogenic ${ }^{3}$ He production rates in apatite, zircon and pyroxene inferred from Bonneville flood erosional surfaces. Quat. Geochronol. 6, 10-21. http://dx.doi.org/10.1016/j.quageo.2010.03.005.

Barrows, T.T., Hope, G.S., Prentice, M.L., Fifield, L.K., Tims, S.G., 2011. Late Pleistocene glaciation of the Mt Giluwe volcano, Papua New Guinea. Quat. Sci. Rev. 30 (19), 2676-2689.

Balco, G., Briner, J., Finkel, R.C., Rayburn, J.A., Ridge, J.C., Schaefer, J.M., 2009 Regional beryllium-10 production rate calibration for late-glacial northeastern North America. Quat. Geochronol. 4, 93-107. http://dx.doi.org/10.1016/ j.quageo.2008.09.001.

Balco, G., Stone, J.O., Lifton, N.A., Dunai, T.J., 2008. A complete and easily accessible means of calculating surface exposure ages or erosion rates from ${ }^{10} \mathrm{Be}$ and ${ }^{26} \mathrm{~A}$ measurements. Quat. Geochronol. 3, 174-195. http://dx.doi.org/10.1016/ j.quageo.2007.12.001.

Blard, P.-H., Bourlès, D., Lavé, J., Pik, R., 2006. Applications of ancient cosmic-ray exposures: theory, techniques and limitations. Quat. Geochronol. 1, 59-73. http://dx.doi.org/10.1016/j.quageo.2006.06.003.

Blard, P.-H., Farley, K.A., 2008. The influence of radiogenic ${ }^{4} \mathrm{He}$ on cosmogenic ${ }^{3} \mathrm{He}$ determinations in volcanic olivine and pyroxene. Earth Planet. Sci. Lett. 276 20-29. http://dx.doi.org/10.1016/j.epsl.2008.09.003.

Blard, P.-H., Lavé, J., Sylvestre, F., Placzek, C.J., Claude, C., Galy, V., Condom, T., Tibari, B., 2013. Cosmogenic ${ }^{3} \mathrm{He}$ production rate in the high tropical Andes (3800 m, 20 S): implications for the local last glacial maximum. Earth Planet. Sci. Lett. 377, 260-275.

Blard, P.H., Lavé, J., Pik, R., Wagnon, P., Bourlès, D., 2007. Persistence of full glacial conditions in the central Pacific until 15,000 years ago. Nature 449 (7162), 591-594.

Blard, P.H., Lave, J., Farley, K.A., Ramirez, V., Jimenez, N., Martin, L.C.P., Charreau, J. Tibari, B., Fornari, M., 2014. Progressive glacial retreat in the Southern Altiplano (Uturuncu volcano, $22^{\circ} \mathrm{S}$ ) between 65 and 14 ka constrained by cosmogenic ${ }^{3} \mathrm{He}$ dating. Quat. Res. 82, 209-221. http://dx.doi.org/10.1016/j.yqres.2014.02.002.

Blard, P.H., Lave, J., Pik, R., Quidelleur, X., Bourles, D., Kieffer, G., 2005. Fossil cosmogenic ${ }^{3} \mathrm{He}$ record from $\mathrm{K}-\mathrm{Ar}$ dated basaltic flows of Mount Etna volcano $\left(\right.$ Sicily, $\left.38^{\circ} \mathrm{N}\right)$ : evaluation of a new paleoaltimeter. Earth Planet. Sci. Lett. 236, 613-631. http://dx.doi.org/10.1016/j.epsl.2005.05.028.

Blard, P.H., Pik, R., Lave, J., Bourles, D., Burnard, P., Yokochi, R., Marty, B., Trusdell, F., 2006. Cosmogenic ${ }^{3} \mathrm{He}$ production rates revisited from evidences of grain size dependent release of matrix-sited helium. Earth Planet. Sci. Lett. 247, 222-234. http://dx.doi.org/10.1016/j.epsl.2006.05.012.

Borchers, B., Marrero, S., Balco, G., Caffee, M., Goehring, B., Lifton, N., Nishiizumi, K., Phillips, F., Schaefer, J., Stone, J., 2016. Geological calibration of spallation production rates in the CRONUS-Earth project. Quat. Geochronol. 31, 188-198. http://dx.doi.org/10.1016/j.quageo.2015.01.009.

Braucher, R., Bourlès, D., Merchel, S., Vidal Romani, J., Fernadez-Mosquera, D. Marti, K., Léanni, L., Chauvet, F., Arnold, M., Aumaître, G., Keddadouche, K., 2013. Determination of muon attenuation lengths in depth profiles from in situ produced cosmogenic nuclides. Nucl. Instrum. Methods Phys. Res. Sect. B Beam Interact. Mater. Atoms 294, 484-490. http://dx.doi.org/10.1016 j.nimb.2012.05.023.

Braucher, R., Merchel, S., Borgomano, J., Bourlès, D.L., 2011. Production of cosmogenic radionuclides at great depth: a multi element approach. Earth Planet. Sci. Lett. 309, 1-9. http://dx.doi.org/10.1016/j.epsl.2011.06.036.

Briner, J., Overeem, I., Miller, G., Finkel, R., 2007. The deglaciation of Clyde Inlet, northeastern Baffin Island, Arctic Canada. J. Ouat. Sci. 22, 223-232.

Briner, J.P., Young, N.E., Goehring, B.M., Schaefer, J.M., 2012. Constraining Holocene $10 \mathrm{Be}$ production rates in Greenland. J. Quat. Sci. 27, 2-6. http://dx.doi.org/ $10.1002 /$ jqs.1562.

Brown, E.T., Stallard, R.F., Larsen, M.C., Raisbeck, G.M., Yiou, F., 1995. Denudation rates determined from the accumulation of in situ produced 10Be in the Luquillo experimental forest, Puerto-Rico. Earth Planet. Sci. Lett. 129, 193-202.

Cerling, T., Craig, H., 1994. Cosmogenic ${ }^{3} \mathrm{He}$ production rates from $39^{\circ} \mathrm{N}$ to $46^{\circ} \mathrm{N}$ latitude, western USA and France. Geochim. Cosmochim. Acta 58, 249-255.

Claude, A., Ivy-Ochs, S., Kober, F., Antognini, M., Salcher, B., Kubik, P.W., 2014. The chironico landslide (Valle Leventina, southern Swiss Alps): age and evolution. Swiss J. Geosci. 107, 273-291. http://dx.doi.org/10.1007/s00015-014-0170-z.

Delunel, R., Blard, P.-H., Martin, L.C.P., Nomade, S., Schlunegger, F., 2016. Long term low latitude and high elevation cosmogenic ${ }^{3} \mathrm{He}$ production rate inferred from a $107 \mathrm{ka}$-old lava flow in northern Chile; $22^{\circ} \mathrm{S}-3400$ ma.s.l. Geochim. Cosmochim. Acta 184, 71-87. http://dx.doi.org/10.1016/j.gca.2016.04.023.

Desilets, D., Zreda, M., 2003. Spatial and temporal distribution of secondary cosmicray nucleon intensities and applications to in situ cosmogenic dating. Earth Planet. Sci. Lett. 206, 21-42. http://dx.doi.org/10.1016/S0012-821X(02)01088-9.

Desilets, D., Zreda, M., Prabu, T., 2006. Extended scaling factors for in situ cosmogenic nuclides: new measurements at low latitude. Earth Planet. Sci. Lett. 246 265-276. http://dx.doi.org/10.1016/j.epsl.2006.03.051.

Douglass, D.C., Singer, B.S., Kaplan, M.R., Mickelson, D.M., Caffee, M.W., 2006 Cosmogenic nuclide surface exposure dating of boulders on last-glacial and late-glacial moraines, Lago Buenos Aires, Argentina: interpretive strategies and paleoclimate implications. Quat. Geochronol. 1, 43-58. http://dx.doi.org/ 10.1016/j.quageo.2006.06.001.

Dunai, T., 2001. Influence of secular variation of the geomagnetic field on production rates of in situ produced cosmogenic nuclides. Earth Planet. Sci. Lett. 193, 197-212. http://dx.doi.org/10.1016/S0012-821X(01)00503-9.

Dunai, T.J., Wijbrans, J.R., 2000. Long-term cosmogenic ${ }^{3} \mathrm{He}$ production rates (152 ka - $1.35 \mathrm{Ma}$ ) from ${ }^{40} \mathrm{Ar} /{ }^{39} \mathrm{Ar}$ dated basalt flows at $29^{\circ} \mathrm{N}$ latitude. Earth Planet. Sci. Lett. 176, 147-156.

Dunne, J., Elmore, D., Muzikar, P., 1999. Scaling factors for the rates of production of cosmogenic nuclides for geometric shielding and attenuation at depth on sloped surfaces. Geomorphology 27, 3-11. http://dx.doi.org/10.1016/S0169555X(98)00086-5.

Elsasser, W.M., Ney, E.P., Wenckler, I.R., 1956. Cosmic ray intensity and geomagnetism. Nature 178, 1226-1227.

Farber, D.L., Hancock, G.S., Finkel, R.C., Rodbell, D.T., 2005. The age and extent of tropical alpine glaciation in the Cordillera Blanca, Peru. J. Quat. Sci. 20, 759-776. http://dx.doi.org/10.1002/jqs.994.

Fenton, C.R., Hermanns, R.L., Blikra, L.H., Kubik, P.W., Bryant, C., Niedermann, S., Meixner, A., Goethals, M.M., 2011. Regional ${ }^{10}$ Be production rate calibration for the past 12 ka deduced from the radiocarbon-dated Grøtlandsura and Russenes rock avalanches at $69^{\circ}$ N. Nor. Quat. Geochronol. 6, 437-452. http://dx.doi.org/ 10.1016/j.quageo.2011.04.005.

Fenton, C.R., Mark, D.F., Barfod, D.N., Niedermann, S., Goethals, M.M., Stuart, F.M., 2013. ${ }^{40} \mathrm{Ar} /{ }^{39} \mathrm{Ar}$ dating of the SP and Bar Ten lava flows $\mathrm{AZ}$, USA: Laying the foundation for the SPICE cosmogenic nuclide production-rate calibration project. Quat. Geochronol. 18, 158-172. http://dx.doi.org/10.1016 j.quageo.2013.01.007.

Fenton, C.R., Niedermann, S., 2014. Surface exposure dating of young basalts (1-200 $\mathrm{ka}$ ) in the San Francisco volcanic field (Arizona, USA) using cosmogenic ${ }^{3} \mathrm{He}$ and ${ }^{21}$ Ne. Quat. Geochronol. 19, 87-105. http://dx.doi.org/10.1016/ j.quageo.2012.10.003.

Finlay, C.C., Maus, S., Beggan, C.D., Bondar, T.N., Chambodut, A., Chernova, T.A., Chulliat, A., Golovkov, V.P., Hamilton, B., Hamoudi, M., Holme, R., Hulot, G., Kuang, W., Langlais, B., Lesur, V., Lowes, F.J., Lühr, H., Macmillan, S., Mandea, M. McLean, S., Manoj, C., Menvielle, M., Michaelis, I., Olsen, N., Rauberg, J., Rother, M., Sabaka, T.J., Tangborn, A., Tøffner-Clausen, L., Thébault, E., Thomson, A.W.P., Wardinski, I., Wei, Z., Zvereva, T.I., 2010. International geomagnetic reference field: the eleventh generation. Geophys. J. Int. 183, 1216-1230. http://dx.doi.org/10.1111/j.1365-246X.2010.04804.x.

Foeken, J.P.T., Stuart, F.M., Mark, D.F., 2012. Long-term low latitude cosmogenic ${ }^{3} \mathrm{He}$ production rate determined from a 126 ka basalt from Fogo, Cape Verdes. Earth Planet. Sci. Lett. 359-360, 14-25. http://dx.doi.org/10.1016/j.epsl.2012.10.005.

Goehring, B.M., Kurz, M.D., Balco, G., Schaefer, J.M., Licciardi, J., Lifton, N., 2010. A reevaluation of in situ cosmogenic ${ }^{3} \mathrm{He}$ production rates. Quat. Geochronol. 5 , 410-418. http://dx.doi.org/10.1016/j.quageo.2010.03.001.

Goehring, B.M., Lohne, Ø.S., Mangerud, J., Svendsen, J.I., Gyllencreutz, R., Schaefer, J., Finkel, R., 2012. Late glacial and holocene ${ }^{10} \mathrm{Be}$ production rates for western Norway. J. Quat. Sci. 27, 89-96. http://dx.doi.org/10.1002/jqs.1517.

Gosse, J.C., Klein, J., Lawn, B., Middleton, R., Evenson, E.B., 1995. Beryllium-10 dating of the duration and retreat of the last pinedale glacial sequence. Science 268 , 1329-1333. http://dx.doi.org/10.1126/science.268.5215.1329.

Gosse, J.C., Phillips, F.M., 2001. Terrestrial in situ cosmogenic nuclides: theory and application. Quat. Sci. Rev. 20, 1475-1560. http://dx.doi.org/10.1016/S02773791(00)00171-2.

Guyodo, Y., Valet, J., 1999. Global changes in intensity of the Earth's magnetic field during the past 800 kyr. Nature 399, 249-252. http://dx.doi.org/10.1038/20420.

Heisinger, B., Lal, D., Jull, A.J.T., Kubik, P., Ivy-Ochs, S., Knie, K., Nolte, E., 2002a. Production of selected cosmogenic radionuclides by muons: 2. Capture of negative muons. Earth Planet. Sci. Lett. 200, 357-369.

Heisinger, B., Lal, D., Jull, A.J.T., Kubik, P., Ivy-Ochs, S., Neumaier, S., Knie, K., Lazarev, V., Nolte, E., 2002b. Production of selected cosmogenic radionuclides by muons; 1. Fast muons. Earth Planet. Sci. Lett. 200, 345-355.

Heyman, J., 2014. Paleoglaciation of the tibetan plateau and surrounding mountains based on exposure ages and ELA depression estimates. Quat. Sci. Rev. 91, 30-41. http://dx.doi.org/10.1016/j.quascirev.2014.03.018.

Jomelli, V., Favier, V., Vuille, M., Braucher, R., Martin, L., Blard, P.-H., Colose, C., Brunstein, D., He, F., Khodri, M., Bourlès, D.L., Leanni, L., Rinterknecht, V., Grancher, D., Francou, B., Ceballos, J.L., Fonseca, H., Liu, Z., Otto-Bliesner, B.L., 2014. A major advance of tropical Andean glaciers during the Antarctic cold reversal. Nature 513, 224-228. http://dx.doi.org/10.1038/nature13546.

Kaplan, M.R., Strelin, J.A., Schaefer, J.M., Denton, G.H., Finkel, R.C., Schwartz, R., Putnam, A.E., Vandergoes, M.J., Goehring, B.M., Travis, S.G., 2011. In-situ cosmogenic 10Be production rate at Lago Argentino, Patagonia: implications for late-glacial climate chronology. Earth Planet. Sci. Lett. 309, 21-32. http:// dx.doi.org/10.1016/j.epsl.2011.06.018.

Kelly, M.A., Lowell, T.V., Applegate, P.J., Phillips, F.M., Schaefer, J.M., Smith, C.A., Kim, H., Leonard, K.C., Hudson, A.M., 2015. A locally calibrated, late glacial ${ }^{10} \mathrm{Be}$ production rate from a low-latitude, high-altitude site in the Peruvian Andes. Quat. Geochronol. 26, 70-85. http://dx.doi.org/10.1016/j.quageo.2013.10.007.

Kim, K.J., Englert, P.A.J., 2004. Profiles of in situ ${ }^{10} \mathrm{Be}$ and ${ }^{26} \mathrm{Al}$ at great depths at the Macraes Flat, East Otago, New Zealand. Earth Planet. Sci. Lett. 223, 113-126. http://dx.doi.org/10.1016/j.epsl.2004.04.006.

Korte, M., Donadini, F., Constable, C.G., 2009. Geomagnetic field for 0-3 ka: 2. A new series of time-varying global models. Geochem. Geophys. Geosystems 10 (Issue 6). http://dx.doi.org/10.1029/2008GC002297. 
Korte, M., Genevey, A., Constable, C.G., Frank, U., Schnepp, E., 2005. Continuous geomagnetic field models for the past 7 millennia: 1 . A new global data compilation. Geochem. Geophys. Geosystems 6. http://dx.doi.org/10.1029/ 2004GC000800.

Kubik, P.W., Ivy-Ochs, S., Masarik, J., Frank, M., Schlüchter, C., $1998 .{ }^{10} \mathrm{Be}$ and ${ }^{26} \mathrm{Al}$ production rates deduced from an instantaneous event within the dendrocalibration curve, the landslide of Köfels, Ötz Valley, Austria. Earth Planet. Sci. Lett. 161, 231-241. http://dx.doi.org/10.1016/S0012-821X(98)00153-8.

Kurz, M., Colodner, D., Trull, T.W., Moore, R.B., O'Brien, K., 1990. Cosmic ray exposure dating with in situ produced cosmogenic ${ }^{3} \mathrm{He}$ : results from young Hawaiian lava flows. Earth Planet. Sci. Lett. 97, 177-189.

Laj, C., Kissel, C., Beer, J., 2004. High resolution global Paleointensity Stack since 75 kyr (GLOPIS-75) calibrated to Absolute values. Timescales Paleomagn. F. Geophys. Monogr. Ser. 145, 255-265. http://dx.doi.org/10.1029/145GM19.

Lal, D., 1991. Cosmic ray labeling of erosion surfaces: in situ nuclide production rates and erosion models. Earth Planet. Sci. Lett. 104, 424-439. http://dx.doi.org/ 10.1016/0012-821X(91)90220-C.

Licciardi, J.M., Kurz, M.D., Clark, P., Brook, E., 1999. Calibration of cosmogenic ${ }^{3} \mathrm{He}$ production rates from Holocene lava flows in Oregon, USA, and effects of the Earth's magnetic field. Earth Planet. Sci. Lett. 172, 261-271. http://dx.doi.org/ 10.1016/S0012-821X(99)00204-6.

Licciardi, J.M., Schaefer, J.M., Taggart, J.R., Lund, D.C., 2009. Holocene glacier fluctuations in the Peruvian Andes indicate northern climate linkages. Science 325, 1677-1679. http://dx.doi.org/10.1126/science.1175010.

Licciardi, J., Kurz, M.D., Curtice, J.M., 2006. Cosmogenic ${ }^{3}$ He production rates from Holocene lava flows in Iceland. Earth Planet. Sci. Lett. 246, 251-264.

Lifton, N.A., 2016. Implications of two Holocene time-dependent geomagnetic models for cosmogenic nuclide production rate scaling. Earth Planet. Sci. Lett. 433, 257-268. http://dx.doi.org/10.1016/j.epsl.2015.11.006.

Lifton, N.A., Bieber, J.W., Clem, J.M., Duldig, M.L., Evenson, P., Humble, J.E., Pyle, R., 2005. Addressing solar modulation and long-term uncertainties in scaling secondary cosmic rays for in situ cosmogenic nuclide applications. Earth Planet. Sci. Lett. 239, 140-161. http://dx.doi.org/10.1016/j.epsl.2005.07.001.

Lifton, N.A., Caffee, M., Finkel, R., Marrero, S., Nishiizumi, K., Phillips, F.M. Goehring, B., Gosse, J., Stone, J., Schaefer, J., Theriault, B., Jull, A.J.T., Fifield, K., 2015. In situ cosmogenic nuclide production rate calibration for the CRONUSEarth project from lake Bonneville, Utah, shoreline features. Quat. Geochronol. 26, 56-69. http://dx.doi.org/10.1016/j.quageo.2014.11.002.

Lifton, N.A., Sato, T., Dunai, T.J., 2014. Scaling in situ cosmogenic nuclide production rates using analytical approximations to atmospheric cosmic-ray fluxes. Earth Planet. Sci. Lett. 386, 149-160. http://dx.doi.org/10.1016/j.epsl.2013.10.052.

Lifton, N.A., Smart, D.F., Shea, M.A., 2008. Scaling time-integrated in situ cosmogenic nuclide production rates using a continuous geomagnetic model. Earth Planet. Sci. Lett. 268, 190-201.

Lupker, M., Blard, P.H., Lavé, J., France-Lanord, C., Leanni, L., Puchol, N., Charreau, J., Bourlès, D., 2012. ${ }^{10} \mathrm{Be}$-derived Himalayan denudation rates and sediment budgets in the Ganga basin. Earth Planet. Sci. Lett. 333-334, 146-156. http:// dx.doi.org/10.1016/j.epsl.2012.04.020.

Ma, X., Li, Y., Bourgeois, M., Caffee, M., Elmore, D., Granger, D., Muzikar, P., Smith, P., 2007. WebCN: a web-based computation tool for in situ-produced cosmogenic nuclides. Nucl. Instrum. Methods Phys. Res. Sect. B Beam Interact. Mater. Atoms 259, 646-652. http://dx.doi.org/10.1016/j.nimb.2007.01.303.

Marrero, S.M., Phillips, F.M., Borchers, B., Lifton, N., Aumer, R., Balco, G., 2016. Cosmogenic nuclide systematics and the CRONUScalc program. Quat. Geochronol. 31, 160-187. http://dx.doi.org/10.1016/j.quageo.2015.09.005.

Martin, L.C.P., Blard, P.-H., Lavé, J., Braucher, R., Lupker, M., Condom, T., Charreau, J., Mariotti, V., Davy, E., 2015. In situ cosmogenic ${ }^{10}$ Be production rate in the High Tropical Andes. Quat. Geochronol. 30, 54-68. http://dx.doi.org/10.1016/ j.quageo.2015.06.012.

Muscheler, R., Beer, J., Kubik, P.W., Synal, H.-A., 2005. Geomagnetic field intensity during the last 60,000 years based on ${ }^{10} \mathrm{Be}$ and ${ }^{36} \mathrm{Cl}$ from the Summit ice cores and ${ }^{14} \mathrm{C}$. Quat. Sci. Rev. 24, 1849-1860. http://dx.doi.org/10.1016/ j.quascirev.2005.01.012.

N.O.A.A, 1976. U.S. Standard Atmosphere. US Gov. Print. Off.

Nishiizumi, K., Imamura, M., Caffee, M.W., Southon, J.R., Finkel, R.C., McAninch, J. 2007. Absolute calibration of ${ }^{10} \mathrm{Be}$ AMS standards. Nucl. Instrum. Methods Phys. Res. Sect. B Beam Interact. Mater. Atoms 258, 403-413. http://dx.doi.org/ 10.1016/j.nimb.2007.01.297.

Nishiizumi, K., Winterer, E.L., Kohl, C.P., Klein, J., Middleton, R., Lal, D., Arnold, J.R., 1989. Cosmic ray production rates of ${ }^{10} \mathrm{Be}$ and ${ }^{26} \mathrm{Al}$ in quartz from glacially polished rocks. J. Geophys. Res. 94, 17907. http://dx.doi.org/10.1029/ JB094iB12p17907.

Obrochta, S.P., Yokoyama, Y., Morén, J., Crowley, T.J., 2014. Conversion of GISP2based sediment core age models to the GICCO5 extended chronology. Quat. Geochronol. 20,1-7. http://dx.doi.org/10.1016/j.quageo.2013.09.001.

Palumbo, L., Benedetti, L., Bourlès, D., Cinque, A., Finkel, R., 2004. Slip history of the Magnola fault (Apennines, Central Italy) from ${ }^{36} \mathrm{Cl}$ surface exposure dating: evidence for strong earthquakes over the Holocene. Earth Planet. Sci. Lett. 225, 163-176. http://dx.doi.org/10.1016/j.epsl.2004.06.012.

Pavón-Carrasco, F.J., Osete, M.L., Torta, J.M., De Santis, A., 2014. A geomagnetic field model for the Holocene based on archaeomagnetic and lava flow data. Earth Planet. Sci. Lett. 388, 98-109.

Peirce, B., 1852. Criterion for the rejection of doubtful observations. Astronomical J. 2 (45), 161-163.
Phillips, F.M., Kelly, M.A., Hudson, A.M., Stone, J.O.H., Schaefer, J., Marrero, S.M. Fifield, L.K., Finkel, R., Lowell, T., 2016. CRONUS-earth calibration samples from the Huancané ii moraines, Quelccaya ice cap. Peru. Quat. Geochronol. 31, 220-236. http://dx.doi.org/10.1016/j.quageo.2015.10.005.

Phillips, F.M., Stone, W.D., Fabryka-Martin, J.T., 2001. An improvement approach to calculating low-energy cosmic-ray neutron fluxes near the land/atmosphere interface. Chem. Geol. 175, 689-701. http://dx.doi.org/10.1016/S0009-2541(00) 00329-6.

Poreda, R.J., Cerling, T.E., 1992. Cosmogenic neon in recent lavas from the. West. United States. Geophys. Res. Lett. 19, 1863-1866.

Putnam, A.E., Schaefer, J.M., Barrell, D.J.A., Vandergoes, M., Denton, G.H., Kaplan, M.R., Finkel, R.C., Schwartz, R., Goehring, B.M., Kelley, S.E., 2010. In situ cosmogenic ${ }^{10} \mathrm{Be}$ production-rate calibration from the Southern Alps, New Zealand. Quat. Geochronol. 5, 392-409. http://dx.doi.org/10.1016/ j.quageo.2009.12.001.

Putnam, A.E., Schaefer, J.M., Denton, G.H., Barrell, D.J.A., Andersen, B.G. Koffman, T.N.B., Rowan, A.V., Finkel, R.C., Rood, D.H., Schwartz, R., Vandergoes, M.J., Plummer, M.A., Brocklehurst, S.H., Kelley, S.E., Ladig, K.L., 2013. Warming and glacier recession in the Rakaia valley, southern Alps of New Zealand, during Heinrich Stadial 1. Earth Planet. Sci. Lett. 382, 98-110. http:// dx.doi.org/10.1016/j.epsl.2013.09.005.

Reimer, P., Bard, E., Bayliss, A., Beck, J., Blackwell, P., Ramsey, C., Buck, C., Cheng, H., Edwards, R., Friedrich, M., Grootes, P., Guilderson, T., Haflidason, H., Hajdas, I., Hatte, C., Heaton, T., Hoffmann, D., Hogg, A., Hughen, K., Kaiser, K., Kromer, B. Manning, S., Niu, M., Reimer, R., Richards, D., Scott, E., Southon, J., Staff, R. Turney, C., van der Plicht, J., 2013. IntCal13 and Marine13 radiocarbon age calibration curves 0-50,000 Years cal BP. Radiocarbon 55, 1869-1887. http:// dx.doi.org/10.2458/azu_js_rc.55.16947.

Riebe, C.S., Kirchner, J.W., Granger, D.E., Finkel, R.C., 2000. Erosional equilibrium and disequilibrium in the Sierra Nevada, inferred from cosmogenic ${ }^{26} \mathrm{Al}$ and ${ }^{10} \mathrm{Be}$ in alluvial sediment. Geology 28, 803-806.

Ritz, J.F., Bourlès, D.L., Philip, H., Raisbeck, G.M., Yiou, F., Enkhtuvshin, B., 1995. Slip rates along active faults estimated with cosmic-ray- exposure dates: application to the Bogd fault, Gobi-Alta, Mongolia. Geology 23, 1019-1022.

Ross, S., 2003. Peirce's criterion for the elimination of suspect experimental data. J. Eng. Technol. 20 (2), 1-12.

Sandstrom, A.E., Pomerantz, M.A., Gronkvist, B.O., 1962. Latitude effect and atmospheric attenuation of the cosmic ray nucleon component. Tellus 14, 356-362.

Sato, T., Yasuda, H., Niita, K., Endo, A., Sihver, L., 2008. Development of PARMA: PHITS-based analytical radiation model in the atmosphere. Radiat. Res. 170 244-259. http://dx.doi.org/10.1667/RR1094.1.

Schimmelpfennig, I., Williams, A., Pik, R., Burnard, P., Niedermann, S., Finkel, R. Schneider, B., Benedetti, L., 2011. Inter-comparison of cosmogenic in-situ ${ }^{3} \mathrm{He}$, ${ }^{21} \mathrm{Ne}$ and ${ }^{36} \mathrm{Cl}$ at low latitude along an altitude transect on the SE slope of Kilimanjaro volcano ( ${ }^{\circ} \mathrm{S}$, Tanzania). Quat. Geochronol. 6, 425-436. http:// dx.doi.org/10.1016/j.quageo.2011.05.002.

Smith, J.A., Seltzer, G.O., Farber, D.L., Rodbell, D.T., Finkel, R.C., 2005. Early local last glacial maximum in the tropical Andes. Science 308, 678-681. http:/ dx.doi.org/10.1126/science.1107075.

Staiger, J., Gosse, J., Toracinta, R., Oglesby, B., Fastook, J., Johnson, J.V., 2007. Atmospheric scaling of cosmogenic nuclide production: climate effect. J. Geophys. Res. Solid Earth 112,1-8. http://dx.doi.org/10.1029/2005JB003811.

Stone, J.O., 2000. Air pressure and cosmogenic isotope production. J. Geophys. Res. 105, 23753. http://dx.doi.org/10.1029/2000JB900181.

Stormer, C., 1955. The Polar Aurora. Oxford University Press.

Stroeven, A.P., Heyman, J., Fabel, D., Björck, S., Caffee, M.W., Fredin, O., Harbor, J.M., 2015. A new Scandinavian reference 10Be production rate. Quat. Geochronol. 29, 105-115. http://dx.doi.org/10.1016/j.quageo.2015.06.011.

Svensson, a., Andersen, K.K., Bigler, M., Clausen, H.B., Dahl-Jensen, D., Davies, S.M. Johnsen, S.J., Muscheler, R., Parrenin, F., Rasmussen, S.O., Rothlisberger, R., Seierstad, I., Steffensen, J.P., Vinther, B.M., 2008. A 60000 Year Greenland Stratigraphic Ice Core Chronology, pp. 47-57. http://dx.doi.org/10.5194/cpd-31235-2007.

Uppala, S.M., KÅllberg, P.W., Simmons, A.J., Andrae, U., Bechtold, V.D.C., Fiorino, M., Gibson, J.K., Haseler, J., Hernandez, A., Kelly, G.A., Li, X., Onogi, K., Saarinen, S., Sokka, N., Allan, R.P., Andersson, E., Arpe, K., Balmaseda, M.A., Beljaars, A.C.M. Van De Berg, L., Bidlot, J., Bormann, N., Caires, S., Chevallier, F., Dethof, A., Dragosavac, M., Fisher, M., Fuentes, M., Hagemann, S., Hólm, E., Hoskins, B.J., Isaksen, L., Janssen, P.A.E.M., Jenne, R., Mcnally, A.P., Mahfouf, J.-F., Morcrette, J.J., Rayner, N.A., Saunders, R.W., Simon, P., Sterl, A., Trenberth, K.E., Untch, A., Vasiljevic, D., Viterbo, P., Woollen, J., 2005. The ERA-40 re-analysis. Q. J. R. Meteorol. Soc. 131, 2961-3012. http://dx.doi.org/10.1256/qj.04.176.

Valet, J.-P., Meynadier, L., Guyodo, Y., 2005. Geomagnetic dipole strength and reversal rate over the past two million years. Nature 435, 802-805.

Vermeesch, P., 2007. CosmoCalc: an Excel add-in for cosmogenic nuclide calculations. Geochem. Geophys. Geosystems 8, 1-14. http://dx.doi.org/10.1029/ 2006GC001530.

Wittmann, H., von Blanckenburg, F., 2009. Cosmogenic nuclide budgeting of floodplain sediment transfer. Geomorphology 109, 246-256. http://dx.doi.org/ 10.1016/j.geomorph.2009.03.006.

Yang, S., Odah, H., Shaw, J., 2000. Variations in the geomagnetic dipole moment over the last 12000 years. Geophys. J. Int. 1982, 158-162. http://dx.doi.org/10.1046 j.1365-246x.2000.00011.x.

York, D., 1966. Least-squares fitting of a straight line. Can. J. Phys. 44, 1079-1086. 
Young, N.E., Schaefer, J.M., Briner, J.P., Goehring, B.M., 2013. A ${ }^{10}$ Be production-rate calibration for the Arctic. J. Quat. Sci. 28, 515-526. http://dx.doi.org/10.1002/ jqs.2642.

Ziegler, L.B., Constable, C.G., Johnson, C.L., Tauxe, L., 2011. PADM2M: a penalized maximum likelihood model of the 0-2 Ma palaeomagnetic axial dipole moment. Geophys. J. Int. 184, 1069-1089. http://dx.doi.org/10.1111/j.1365246X.2010.04905.X.

Zweck, C., Zreda, M., Anderson, K.M., Bradley, E., 2012. The theoretical basis of ACE an Age Calculation Engine for cosmogenic nuclides. Chem. Geol. 291, 199-205. http://dx.doi.org/10.1016/j.chemgeo.2011.10.005. 